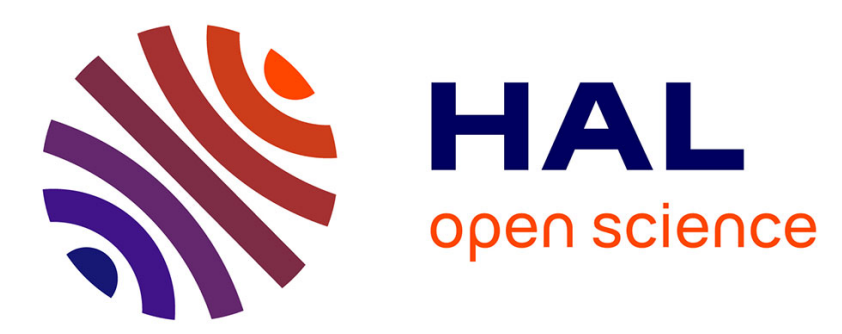

\title{
On a new harmonic selection technique for harmonic balance method
}

Aurelien Grolet, Fabrice Thouverez

\section{To cite this version:}

Aurelien Grolet, Fabrice Thouverez. On a new harmonic selection technique for harmonic balance method. Mechanical Systems and Signal Processing, 2012, 30, pp.43-60. 10.1016/j.ymssp.2012.01.024 . hal-02121478

\section{HAL Id: hal-02121478 \\ https://hal.science/hal-02121478}

Submitted on 18 Dec 2019

HAL is a multi-disciplinary open access archive for the deposit and dissemination of scientific research documents, whether they are published or not. The documents may come from teaching and research institutions in France or abroad, or from public or private research centers.
L'archive ouverte pluridisciplinaire HAL, est destinée au dépôt et à la diffusion de documents scientifiques de niveau recherche, publiés ou non, émanant des établissements d'enseignement et de recherche français ou étrangers, des laboratoires publics ou privés. 


\title{
On a new harmonic selection technique for harmonic balance method
}

\author{
Aurélien Grolet*, Fabrice Thouverez \\ École Centrale de Lyon, Laboratoire de Tribologie et Dynamique des Systèmes, 36, avenue Guy de Collongue, 69134 Ecully Cedex, France
}

\begin{abstract}
This paper is intended to present a new harmonic selection technique when solving nonlinear dynamic systems with the harmonic balance method. This technique belongs to the class of method called the adaptive harmonic balance method (AHBM). The harmonic selection is based on the use of a tangent predictor and relies on a stepwise regression procedure that allows for a dynamic management of the number of selected harmonics via an addition or removal procedure. The efficiency of this method relative to the classical harmonic balance method (HBM) is then evaluated through examples; this later step will indicate that AHBM can significantly reduce the number of variables, thus leading to computational time savings without deteriorating solution quality.
\end{abstract}

\section{Introduction}

The harmonic balance method (HBM) enjoys widespread use and is able to treat many types of nonlinearities (contact and friction [11,17], geometric nonlinearities [16], Euler's equation, unsteady aerodynamic [14,8], etc.). HBM consists of identifying periodic solutions in the form of a truncated Fourier series, whose coefficients are estimated by solving a set of nonlinear algebraic equations. The most important parameter when applying this method is the number of selected harmonics used to approximate the solution. Typically, the higher the number of harmonics, the better the solution. For large-sized systems however, adding harmonics can prove very costly in terms of extra computational resources. If the number of nonlinear degrees of freedom (dof) is small compared to the number of linear dofs, then one method to reduce problem size would be to condense the problem into the nonlinear dof [17]. However, if all dofs are nonlinear, this condensation step would no longer be applicable and other methods would need to be used in order to reduce computational resources.

When nonlinear dofs constitute just a subset or when the nonlinearity is distributed spatially, it can be observed that all dofs are not submitted to the same level of nonlinearity; hence, the number of selected harmonics may be overestimated for either linear or weakly nonlinear dofs. This finding implies that some variables of the algebraic system are insignificant since they are either equal or near zero. These excess variables needlessly slow the calculation.

In order to correctly estimate the number of selected harmonics for a given level of accuracy and thereby reduce the size of the set of nonlinear algebraic equations, a number of selection algorithms have been proposed, thus leading to methods known as adaptive harmonic balance methods (or AHBM) [11-14].

The aim of this paper is to review and discuss the available methods for harmonic selection in HBM (Section 2) and to present a new selection method (Section 3). The efficiency of the proposed method will then be demonstrated through a series of three examples (Section 4 ).

\footnotetext{
* Principal Corresponding author. Tel.: +33633895325.

E-mail addresses: aurelien.grolet@ecl2009.ec-lyon.fr (A. Grolet), fabrice.thouverez@ec-lyon.fr (F. Thouverez).
} 


\section{HBM and harmonic selection}

\subsection{HBM principles}

In this section, the principles of HBM will briefly be recalled. Many papers in the literature focus on the method, and readers are referred to the bibliography for further details regarding this method (see in particular $[10,11,16,15]$ ).

Let us consider an $n$-dof dynamic system whose motion equation is given by the following:

$$
\mathbf{M U}+\mathbf{C} \dot{U}+\mathbf{K U}+\boldsymbol{F}_{n l}(\boldsymbol{U}, \dot{U})=\boldsymbol{F}_{e x}
$$

where $\mathbf{M}, \mathbf{C}$ and $\mathbf{K}$ are, a respectively, the mass, damping and stiffness matrices; $\boldsymbol{F}_{n l}$ is the vector of nonlinear forces, and $\boldsymbol{F}_{e x}$ is the vector of external forces, assumed to be periodic with a period $T=2 \pi / \omega$. The solution $\boldsymbol{U}(t)$ of Eq. (1) can then be sought according to the following form:

$$
\boldsymbol{U}(t)=\boldsymbol{U}_{0}+\sum_{k=1}^{N_{h}} \boldsymbol{U}_{k}^{c} \cos (k \omega t)+\boldsymbol{U}_{k}^{s} \sin (k \omega t)
$$

After application of the HBM projections (see Appendix A), the following set of nonlinear algebraic equations is obtained:

$$
\boldsymbol{\Lambda}(\omega) \tilde{\boldsymbol{U}}+\tilde{\boldsymbol{F}}_{n l}=\tilde{\boldsymbol{F}}_{e x}
$$

With $\boldsymbol{\Lambda}$ denoting the dynamic stiffness matrix, $\tilde{\boldsymbol{U}}$ the vector of Fourier coefficients (i.e. the vector of unknowns in the frequency domain), and $\tilde{\boldsymbol{F}}_{n l}$ and $\tilde{\boldsymbol{F}}_{e x}$ the vectors of nonlinear and external forces in the frequency domain. All these vector and matrices are of size $M=n\left(2 N_{h}+1\right)$, where $N_{h}$ is the number of selected harmonics in the Fourier approximation of $\boldsymbol{U}(t)$ (Eq. (2)).

When analytical expression cannot be derived for $\tilde{\boldsymbol{F}}_{n l}$, this vector is computed through the use of an alternate frequency-time (AFT) procedure [4]. The AFT procedure consists of evaluating the temporal evolution of $\boldsymbol{U}(t)$ with an inverse fast Fourier transform (iFFT) of $\tilde{\boldsymbol{U}}$. The nonlinear forces are then computed within the time domain, and lastly the Fourier coefficients $\tilde{\boldsymbol{F}}_{n l}$ are evaluated with an FFT.

Periodic solutions to the system in Eq. (1) are found by solving the nonlinear algebraic system in Eq. (3). When simulations take place over a frequency range $\left[\omega_{1}, \omega_{2}\right]$, one convenient way to solve the system is to use the continuation methods [15,10], which consists of two steps: first, a predictor computes an estimation of the solution for the next frequency point, then, a corrector (often derived from a Newton method) is applied to this prediction to derive the true solution.

\subsection{Existing selection algorithm}

When solving Eq. (3), if the number of retained harmonic $N_{h}$ is too high, then a number $M_{i}$ of unknowns may prove insignificant, as a result of being equal or near zero. Selection algorithms will be introduced to reduce the system size by means of removing the $M_{i}$ insignificant variables.

Selection methods from the literature can be divided into two types: global selection and local selection.

\subsubsection{Global selection}

A global selection consists of setting an equal number of harmonics for all dof; this number is then updated by evaluating a criterion that quantifies the nonlinear contents of nonlinear forces. Depending on the choice of this criterion, two selection methods have already been proposed.

The first example was presented by Laxalde in [12]. The criterion used in this method corresponds to the Fourier error between nonlinear forces in the time domain and the reconstruction of nonlinear forces from the selected Fourier coefficient. This criterion is expressed as follows:

$$
\epsilon\left(N_{h}\right)=\left\|\int_{0}^{2 \pi / \omega} \boldsymbol{F}_{n l}(\boldsymbol{U}, \dot{\boldsymbol{U}})-\left[\boldsymbol{F}_{0}+\sum_{k=1}^{N_{h}} \boldsymbol{F}_{k}^{c} \cos (k \omega t)+\boldsymbol{F}_{k}^{s} \sin (k \omega t)\right] d t\right\|
$$

If the Fourier error exceeds a threshold value, then the number of harmonics is increased for the computation at the next frequency step. Note that the number of harmonics to be added for the purpose of decreasing the Fourier error is an $a$ priori fixed increment to be defined by the user.

The second example of harmonic selection was presented by Jaumouille in [11]. The criterion used is the variation in strain energy with the number of harmonics. Strain energy is first calculated for a number $N_{h}^{0}$ of selected harmonics and then for a number $N_{h}^{0}+1$. The relative difference between these two energies is evaluated as follows:

$$
\epsilon\left(N_{h}^{0}\right)=\frac{\left|S\left(N_{h}^{0}\right)-S\left(N_{h}^{0}+1\right)\right|}{\left|S\left(N_{h}^{0}\right)\right|}
$$

where $S$ stands for the strain energy. The number of selected harmonics is given whenever the relative difference lies below a threshold value. 


\subsubsection{Local selection}

Local selection relies upon the fact that all dof are not exposed to the same level of nonlinearity. Consequently, the number of selected harmonics should be variable with respect to the dof. The local selection method thus consists of finding a number of selected harmonics for each dof. In its construction, this method is a sort of global method extension since the number of selected harmonics varies not just with dof but with frequency as well.

A local selection method had been proposed by Maple in [14,13]. For each dof $d$, the fraction of spectral energy contained in the last selected harmonic of the computed solution is evaluated as follows:

$$
\epsilon\left(N_{h}, d\right)=\frac{\left(U_{N_{h}}^{d, c}\right)^{2}+\left(U_{N_{h}}^{d, s}\right)^{2}}{\left(U_{0}^{d}\right)^{2}+\sum_{k=1}^{N_{h}}\left(U_{k}^{d, c}\right)^{2}+\left(U_{k}^{d, s}\right)^{2}}
$$

If this fraction exceeds a fixed threshold value, then additional harmonics need to be introduced, and the solution is computed once again with this new number of harmonics. Like with global methods, the number of harmonics to be added is determined by an a priori fixed increment.

\subsubsection{Limitations of the previous methods}

The three previous examples of harmonic selection methods all exhibit some limitations: they are incremental and the increment on the number of harmonic to be added is set by the user.

Incremental means that, for a fixed frequency step of the HBM, the harmonics truncation order is increasing incrementally until a stop criterion is satisfied. Consequently, for each iteration of the selection procedure (i.e. for each increase/decrease of the total number of harmonics by the increment), it is necessary to compute a complete nonlinear solution of the system for the fixed frequency step in order to evaluate the respective stop criteria. This procedure allows for precise control over the error (induced by criterion), yet they can be very time consuming due to the many possible computations of nonlinear solutions required for the criteria evaluation. In his thesis [12], Laxalde choose to present his selection method in a non-incremental way, i.e. for a fixed frequency step of the HBM Fourier's error is computed for a given number of harmonics and if this error is high relative to the threshold value, the number of harmonics is increased for the next frequency step. Doing so, Fourier's error can be larger than the threshold value and the method then does not really control Fourier's error. An incremental version of this method can be easily derived.

In the incremental procedures, an important parameter is the increment defining the number of harmonic to be added/ removed from one iteration of the selection procedure to another. This increment is set by users and there is $a$ priori no information on how to choose it. On one hand, if this increment is small, it can result in many iteration for the selection procedure to make the error smaller than the threshold value, thus leading to many nonlinear calculation for a given frequency step. On the other hand, if this increment is too large, insignificant harmonics could be added leading to an unnecessary large system of algebraic equation to be solved. Moreover if the increment is not chosen well, it can lead to premature end of the selection procedure. For instance, in Jaumouillé's method it may happen that the relative difference between $S\left(N_{h}\right)$ and $S\left(N_{h}+\Delta N_{h}\right)$ is zero while the relative difference between $S\left(N_{h}\right)$ and $S\left(N_{h}+2 \Delta N_{h}\right)$ is larger than the threshold value, thus leading to a premature stop of the selection process. This is typically the case if $\Delta N_{h}=1$ and if only if odd harmonics respond, or more generally when a subset of non-consecutive harmonics responds.

The next section presents a new selection procedure which intend to overcome the two limitations exposed before while maintaining the local nature of the selection.

\section{Proposed selection method}

\subsection{Preliminary remarks}

The procedure presented here will start by defining a maximum number of harmonics to be retained and building the corresponding system of HBM equation. Let $N_{h}^{m}$ be the maximum number of harmonics intended to be used in the computation of solutions. In the case where all dof are computed with all $N_{h}^{m}$ harmonics, the HBM equation (Eq. (3)) can be rewritten as follows:

$$
\boldsymbol{f}(\tilde{\boldsymbol{U}}, \omega)=\boldsymbol{\Lambda}(\omega) \tilde{\boldsymbol{U}}+\tilde{\boldsymbol{F}}_{n l}-\tilde{\boldsymbol{F}}_{e x}=\mathbf{0}
$$

which is a system with $M_{m}=n\left(2 N_{h}^{m}+1\right)$ unknowns and $M_{m}$ equations. Let $\boldsymbol{I}_{\boldsymbol{m}}=\left[1 . M_{m}\right]$ be a list of index corresponding to all variables in Eq. (7).

Consider now that we have a selection method that enables defining a reduced vector of unknown called $\tilde{\boldsymbol{U}}_{r}$ through an index vector $\boldsymbol{I}_{\boldsymbol{r}}$ ( of length $M_{r}$ ) corresponding to significant variables only (thus with $M_{r}=$ length $\left.\left(\boldsymbol{I}_{r}\right) \leq \operatorname{length}\left(\boldsymbol{I}_{\boldsymbol{m}}\right)=M_{m}\right)$. The procedure for deriving the index vector $\boldsymbol{I}_{r}$ will be presented in the next section. In this case the system to be solved (also called the reduced system) can be written as

$$
\boldsymbol{f}_{r}\left(\tilde{\boldsymbol{U}}_{r}, \omega\right)=\mathbf{0}
$$

which is a system of $M_{r}\left(\leq M_{m}\right)$ unknowns and $M_{r}$ equations. 
The vector $\tilde{\boldsymbol{U}}_{r}$ in Eq. (8) is derived from the vector $\tilde{\boldsymbol{U}}$ in Eq. (7) by considering its restriction to the variables indexed by $I_{r}$ as follows:

$$
\tilde{\boldsymbol{U}}_{r}=\left.\tilde{\boldsymbol{U}}\right|_{\boldsymbol{I}_{r}}
$$

Conversely the vector $\tilde{\boldsymbol{U}}_{r}$ of Eq. (8) can be transformed into a vector $\tilde{\boldsymbol{U}}^{\text {rec }}$ in Eq. (7) (a reconstituted vector) by considering that the values of the non-selected variables equal zero:

$$
\left.\tilde{\boldsymbol{U}}^{\text {rec }}\right|_{\boldsymbol{I}_{r}}=\tilde{\boldsymbol{U}}_{r} \text { and }\left.\tilde{\boldsymbol{U}}^{\text {rec }}\right|_{\boldsymbol{I}_{m r}}=\mathbf{0} \text { (with } \boldsymbol{I}_{m}=\boldsymbol{I}_{r} \cup \boldsymbol{I}_{m r} \text { ) }
$$

The set of $M_{r}$ equation $\boldsymbol{f}_{r}$ is derived from the set of $M_{m}$ equation $\boldsymbol{f}$ as follows:

$$
\boldsymbol{f}_{r}\left(\tilde{\boldsymbol{U}}_{r}, \omega\right)=\left.\boldsymbol{f}\right|_{\boldsymbol{I}_{r}}\left(\tilde{\boldsymbol{U}}^{r e c}, \omega\right)
$$

In other words, only the $M_{r}$ equations related to the $M_{r}$ variables in $\tilde{\boldsymbol{U}}_{r}$ are retained by considering that the $i$ th equation is related to the $i$ th variable.

Finally, for $1 \leq d \leq n$ we define $\tilde{\boldsymbol{u}}^{d}$ (resp. $\tilde{\boldsymbol{u}}_{r}^{d}$ ) the vector of Fourier coefficients (resp. the reduced vector of Fourier coefficients) corresponding to dof $d$. Vector $\tilde{\boldsymbol{u}}^{d}$ assumes the following form:

$$
\tilde{\boldsymbol{u}}^{d}=\left[u_{0}^{d}, u_{1 c}^{d}, u_{1 s}^{d}, \ldots, u_{N_{h}^{m} c}^{d}, u_{N_{h}^{m} s}^{d}\right]
$$

with $u_{0}$ the continuous component, and $u_{k c}\left(1 \leq k \leq N_{h}^{m}\right)$ [resp. $u_{k s}$ ] the coefficient of $\cos (k \omega t)$ [resp. $\left.\sin (k \omega t)\right]$ in the Fourier development of $\boldsymbol{u}^{d}$.

$\tilde{\boldsymbol{u}}_{r}^{d}$ is associated with the index vector $\boldsymbol{I}_{\boldsymbol{r}}^{d}$ (in relation to $\tilde{\boldsymbol{U}}$ ) and with index vector $\boldsymbol{J}_{\boldsymbol{r}}^{d}$ (in relation to $\tilde{\boldsymbol{u}}^{d}$ ) such that

$$
\tilde{\boldsymbol{u}}_{r}^{d}=\left.\tilde{\boldsymbol{U}}\right|_{\boldsymbol{I}_{r}^{d}}=\left.\tilde{\boldsymbol{u}}^{d}\right|_{\boldsymbol{J}_{r}^{d}}
$$

with

$$
\boldsymbol{I}_{\boldsymbol{r}}^{d} \subset\left[1 \ldots M_{m}\right], \quad \boldsymbol{I}_{\boldsymbol{r}}=\bigcup_{d=1}^{n} \boldsymbol{I}_{\boldsymbol{r}}^{d}, \quad \boldsymbol{J}_{\boldsymbol{r}}^{d} \subset\left[1 \ldots 2 N_{h}^{m}+1\right]
$$

For example, if only the continuous component, the first and the third harmonics (cosine and sine terms) were selected for dof $d$ we would have $\boldsymbol{J}_{\boldsymbol{r}}^{d}=[1,2,3,6,7]$ (see Eq. (12)).

\subsection{Prediction step}

Let us now assume that a solution $\left(\tilde{\boldsymbol{U}}_{r}^{i}, \omega^{i}\right)$ to Eq. (8) has been computed (where $i$ stands for the frequency step), associated with the index vector $\boldsymbol{I}_{\boldsymbol{r}}^{i}$. One estimation of the solution for the next frequency step can be given through the use of a tangent predictor [15]. In using Eq. (7) instead of Eq. (8) to compute this prediction, information could be obtained on the participation of the non-selected harmonics in the solution at the next frequency step. Consequently, the vector considered in the prediction phase will always be of maximum size $M_{m}$. To compute the prediction, the solution $\left(\tilde{\boldsymbol{U}}_{r}^{i}, \omega^{i}\right)$ of Eq. (8) is first transformed into a solution $\left(\tilde{\boldsymbol{U}}^{i}, \omega^{i}\right)$ of Eq. (7) by adding zeros, as described previously in Eq. (10). Then, the predicted vector $\tilde{\boldsymbol{U}}_{p}$ is given by the following:

$$
\tilde{\boldsymbol{U}}_{p}=\tilde{\boldsymbol{U}}^{i}+\Delta \tilde{\boldsymbol{U}}^{i}
$$

with $\Delta \boldsymbol{U}^{i}$ being solution of the following linear system:

$$
\frac{\partial \boldsymbol{f}}{\partial \tilde{\boldsymbol{U}}}\left(\tilde{\boldsymbol{U}}^{i}, \omega^{i}\right) \Delta \tilde{\boldsymbol{U}}^{i}=-\frac{\partial \boldsymbol{f}}{\partial \omega}\left(\tilde{\boldsymbol{U}}^{i}, \omega^{i}\right) \Delta \omega^{i}
$$

In order to apply the selection method to each dof, the predicted vector is decomposed into $n$ subvectors of size $2 N_{h}^{m}+1$ corresponding to the Fourier coefficients of each dof. These subvectors are denoted $\tilde{\boldsymbol{u}}_{p}^{d}$ for $1 \leq d \leq n$ and are organized as described in Eq. (12). In addition, each local predicted vector $\tilde{\boldsymbol{u}}_{p}^{d}$ is associated with an index vector $\boldsymbol{J}_{\boldsymbol{r}}^{i, d}$ which corresponds to variables that have been used in the computation of $\tilde{\boldsymbol{u}}_{r}^{i, d}$ as defined in Eq. (13).

\subsection{Selection criteria}

Let us consider dof $d\left(\tilde{\boldsymbol{u}}_{r}^{i, d}\right)$, which has been computed using the Fourier coefficients associated with $\boldsymbol{J}_{\boldsymbol{r}}^{i, d}$. We can forward the hypothesis that the true solution at the next frequency step $i+1$ does not significantly deviate from the prediction. As a consequence, let us consider that the predicted vector have roughly the same harmonic content as the true solution. The parameter $\epsilon_{k}^{d}$ corresponding to the fraction of spectral energy contains in the harmonic of rank $k$ of the predicted subvector $\tilde{\boldsymbol{u}}_{p}^{d}$ can then be defined as follows:

$$
\left.\epsilon_{k}^{d}=\left[\left(u_{p, k c}^{d}\right)^{2}+\left(u_{p, k s}^{d}\right)^{2}\right)\right] /\left\|\tilde{\boldsymbol{u}}_{p}^{d}\right\|^{2} \quad \text { for } 0 \leq k \leq N_{h}^{m}
$$


Thanks to the index vector $\boldsymbol{J}_{\boldsymbol{r}}^{i, d}$ and the predicted subvector $\tilde{\boldsymbol{u}}_{p}^{d}$, we are able to evaluate the percentage of energy $E_{r}^{i, d}$ that will be retained if we use the Fourier coefficients associated with $\boldsymbol{J}_{\mathbf{r}}^{i, d}$ :

$$
E_{r}^{d}=\sum_{k \in \boldsymbol{H}\left(\boldsymbol{J}_{r}^{i, d}\right)} \epsilon_{k}^{d}
$$

where $\boldsymbol{H}\left(\boldsymbol{J}_{\mathbf{r}}^{i, d}\right)$ is an index vector linking each element in $\boldsymbol{J}_{\boldsymbol{r}}^{i, d}$ to its harmonic number. For example, if $\boldsymbol{J}_{\boldsymbol{r}}^{i, d}=[1,2,3,6,7]$ then $\boldsymbol{H}\left(J_{r}^{i, d}\right)=[0,1,3]$ (the continuous component, the first and the third harmonics are retained). We also define the fraction of residual energy $\rho^{d}$ (i.e. the percentage of energy contained in the non-selected Fourier coefficient) as follows:

$$
\rho^{d}=1-E_{r}^{d}
$$

The notion behind the selection procedure is to asses the fraction of residual energy $\rho^{d}$ compared with two threshold values $\rho_{b}$ and $\rho_{f}$ (with $0 \leq \rho_{b} \leq \rho_{f} \leq 1$ ):

- Case 1: If $\rho^{d} \geq \rho_{f}$, the error committed is too large and Fourier coefficients need to be added in order to reduce error $\rho^{d}$ (through the use of the forward procedure defined hereafter), thus leading to $\operatorname{card}\left(\boldsymbol{J}_{\mathbf{r}}^{i+1, d}\right) \geq \operatorname{card}\left(\boldsymbol{J}_{\mathbf{r}}^{i, d}\right)$.

- Case 2: If $\rho^{d} \leq \rho_{b}$, the error committed is too small and Fourier coefficients can be removed so as to reasonably increase the error $\rho^{d}$ (through the use of the backward procedure defined hereafter), leading to $\operatorname{card}\left(\boldsymbol{J}_{\mathbf{r}}^{i+1, d}\right) \leq \operatorname{card}\left(\boldsymbol{J}_{\mathbf{r}}^{i, d}\right)$.

- Case 3: If $\rho_{b} \leq \rho^{d} \leq \rho_{f}$, then the error committed is at an intermediate level, leading to the supposition that the number of Fourier coefficients is sufficient for the next frequency step, thus leading to $\boldsymbol{J}_{\mathbf{r}}^{i+1, d}=\boldsymbol{J}_{\mathbf{r}}^{i, d}$.

\subsubsection{Forward procedure}

This procedure is implemented for the purpose of adding Fourier coefficients (Case 1) in order to obtain $\rho^{d} \leq \rho_{f}$; it consists of adding the spectral energy contained in the non-selected variable associated with the set $\boldsymbol{J}_{\boldsymbol{m r}}^{i, d}=\left[1: 2 N_{h}^{m}+1\right] \boldsymbol{J}_{\boldsymbol{r}}^{i, d}$ until the residual spectral energy lies below the threshold value $\rho_{f}$. For each non-selected variable of $\tilde{\boldsymbol{u}}_{p}^{d}$ the parameters $\epsilon_{k}^{d}$ are computed and ordered. The variables generating the highest values for $\epsilon_{k}^{d}$ can then be added to the selected variable until $\rho^{d} \leq \rho_{f}$.

\subsubsection{Backward procedure}

This procedure is used to remove Fourier coefficients (Case 2) in order to obtain $\rho_{b} \leq \rho^{k}$. In this case, the procedure consists of removing the spectral energy contained in the selected variables until the spectral residual energy is greater than the threshold value $\rho_{b}$. For each selected variable of $\tilde{\boldsymbol{u}}_{p}^{d}$ the parameters $\epsilon_{k}^{d}\left(k \in \boldsymbol{H}\left(\boldsymbol{J}_{r}^{i, d}\right)\right)$ are computed and ordered. The variables producing the lowest values for $\epsilon_{k}^{d}$ are then removed from the selected variables until $\rho_{b} \leq \rho^{i+1, d}$.

The method described for one dof is then applied to all system dof, which leads to a series of local index vector $\left(J_{\boldsymbol{r}}^{i+1, d}\right)_{1 \leq d \leq n}$ indicating the Fourier coefficients to be retained for each dof $d$ at the next frequency step $i+1$. Afterwards, this series is converted into a series of global index vector $\left(\boldsymbol{I}_{\boldsymbol{r}}^{i+1, d}\right)_{1 \leq d \leq n}$ (see Eq. (13)) that defines the selected variables to be used during the next frequency step. The diagram in Fig. 1 summarizes this harmonic selection algorithm.

Note that when the continuous component of the predicted vector is larger than its harmonics component, the computation of $\epsilon_{k}^{d}$ in Eq. (17) is subjected to rounding error. To overcome these numerical issues, it can be considered that the continuous component is always selected and then the parameters $\epsilon_{k}^{d}$ can be computed without taking into account the continuous component (i.e. by assuming that $\tilde{\boldsymbol{u}}_{p}^{d}$ in Eq. (17) no longer has a continuous component).

\subsection{Remarks and comparison with existing methods}

As explained in Section 2.2, existing selection methods are incremental and add harmonics by increment until the stopping criterion is achieved. At each iteration they will seek information about the next harmonic number, and if the number of harmonics to be retained is not bounded it can grow very large if the stop criteria is never satisfied. In the new method presented here, a (possibly large) maximum number of harmonic $N_{h}^{m}$ is defined at the beginning of the procedure thus defining a maximum set of harmonic in which the selection procedure seeks harmonics to be retained, thus making it non-incremental. The selection method operates independently for each dof and give different set of retained harmonics for each dof thus making it a local type of selection.

The key points of the new method are (a) the use of a tangent prediction over the set of all harmonic (the predicted vector is always of maximum size $M_{m}$ ) which allows to evaluate and sort the participation of all harmonics smaller than $N_{h}^{m}$ at the same time and (b) the hypothesis that the prediction is sufficiently close to the real solution in order to say that the real solution will have the same harmonics support as the prediction. Only a tangent predictor can be used for the prediction, since only this type of predictor can yield informations about non-selected Fourier coefficients through the Jacobian matrix $(\partial \boldsymbol{f} / \partial \tilde{\boldsymbol{U}})\left(\tilde{\boldsymbol{U}}^{i}, \omega^{i}\right)$. Contrary to existing methods, no nonlinear calculations have to be performed in order to evaluate harmonics participation. One limitation of the proposed method appears when the prediction deviates too far from the true solution. However, if the frequency step is small enough, this prediction will be close to the true solution.

As in Maple's procedure, the criteria used to quantify the participation of a harmonic of the predicted vector is the spectral density of energy contained in this harmonic, but here the spectral density is computed for all harmonics smaller than $N_{h}^{m}$, thus giving an overview of each harmonic participation. The threshold value $\rho_{f}$ controlling the forward procedure 


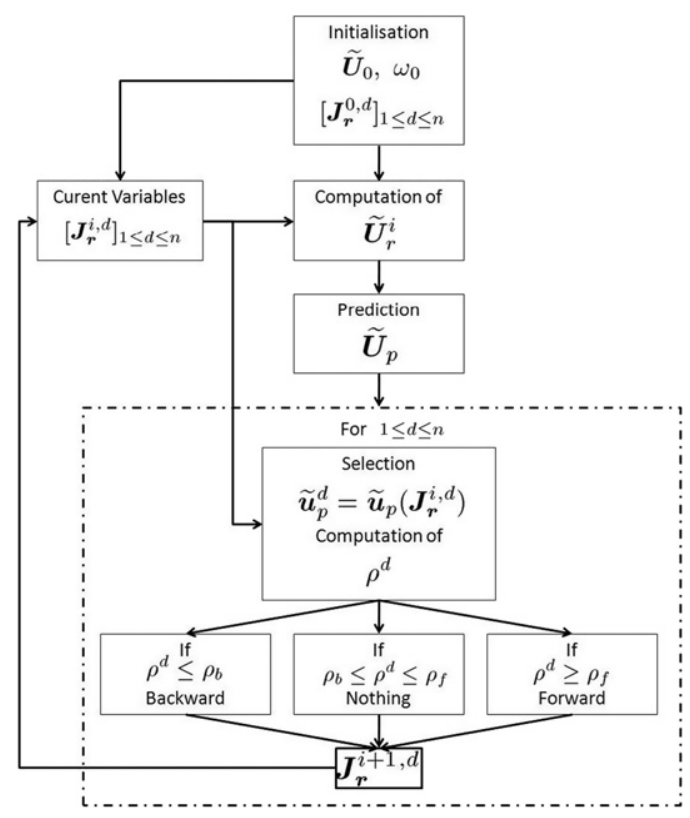

Fig. 1. Description of the harmonic selection process.

must be set at a low level to be able to select a number of Fourier coefficients that ensures good approximation of the solution. The same principle applies to the threshold value $\rho_{b}$ which must also be set low so as not to remove Fourier coefficients too early. As in the existing methods the threshold values $\left(\rho_{f}\right.$ and $\left.\rho_{b}\right)$ must be empirically set by users, yet they have a physical meaning and are related to the percentage of energy that will be retained in the predicted vector. Clearly, if the threshold values are set to zero, all the responding harmonics are selected and the error then equals the error of the classical HBM with $N_{h}^{m}$ harmonics.

The cost of this method lies primarily in the computational time required to establish the predicted vector, which consists of solving a (possibly large) linear system of size $M_{m}$. Yet this time duration is often small when compared with the time required to solve the total set of nonlinear algebraic equations in Eq. (7) using Newton-Raphson algorithm. Moreover, in continuation of the techniques, the computation of this predicted vector is a compulsory step; in this case therefore, the cost of the proposed method is solely determined in the forward or backward procedure employed, and both are inexpensive from a computational standpoint since they only entail computing the $\epsilon_{k}^{d}$ parameters in Eq. (17) and then ordering them.

\section{Examples}

In order to evaluate method efficiency, we will now present three examples featuring different types of nonlinearity. The first one is a small and simple dynamic system with smooth nonlinearity, the second example is a small system with cubic nonlinearity featuring an internal resonance and the third example has been derived from an industrial problem with a non-smooth nonlinearity. A comparison will be drawn with the classical harmonic balance method (considered as the reference method) in order to estimate the benefits of the proposed harmonic selection method.

\subsection{Example 1: A nonlinear system featuring internal resonances}

In order to confirm that the AHBM performs adequately well for many types of nonlinear systems, we will consider in this section the special case of a system with internal resonances. In general terms, internal resonances appear when the frequencies of the linearized system (natural frequencies) are commensurable or nearly commensurable $[1,2]$. However, due to the fact that resonance frequencies change with motion amplitude in nonlinear systems, cases of internal resonances can also appear when natural frequencies are not commensurable (see for example [9]).

Internal resonances are responsible for strong modal interactions and sub-harmonic or super-harmonics resonances. For instance in [1], Nayfeh considered a nonlinear system with cubic nonlinearity and with a 1:3 internal resonance between the first and the second mode of the system. By applying the method of multiple scales, he was able to show that the response could be dominated by the first vibration mode whenever the excitation frequency was positioned near the second natural frequency.

In this example we will re-demonstrated this result with the use of the HBM. We will also demonstrate that the HBM is able to treat the internal resonance case, and that the AHBM proposed herein can reduce the number of harmonics involved, proving its adaptation for these types of systems. 


\subsubsection{System definition}

The system used in this example is the one presented in [1]. It corresponds to a beam of normalized length $l=2$ with one end clamped and the other hinged. Motion equations for the first two vibration modes have been derived from the Galerkin procedure and are given by the following:

$$
\begin{aligned}
& \ddot{u}_{1}+2 \epsilon c_{1} \dot{u}_{1}+\omega_{1}^{2} u_{1}+\epsilon v f_{n l, 1}\left(u_{1}, u_{2}\right)=F_{1} \cos (\Omega t) \\
& \ddot{u}_{2}+2 \epsilon c_{2} \dot{u}_{2}+\omega_{2}^{2} u_{2}+\epsilon v f_{n l, 2}\left(u_{1}, u_{2}\right)=F_{2} \cos (\Omega t)
\end{aligned}
$$

where $\left(u_{1}, u_{2}\right)$ are the amplitude of the first and the second mode of vibration, $\left(\omega_{1}, \omega_{2}\right)$ the first two natural frequencies, $\left(c_{1}, c_{2}\right)$ the modal damping coefficients, $\Omega$ the excitation frequency, and lastly $\left(f_{n l, 1}, f_{n l, 2}\right)$ the nonlinear forces given by

$$
f_{n l, i}\left(u_{1}, u_{2}\right)=\alpha_{i, 1} u_{1}^{3}+\alpha_{i, 2} u_{1}^{2} u_{2}+\alpha_{i, 3} u_{1} u_{2}^{2}+\alpha_{i, 4} u_{2}^{3}, \quad i=1,2
$$

For the remainder of this example we will consider that $\epsilon=10^{-4}$, and $v=1 / 4$. The numerical values for the other parameters in Eqs. (20) and (21) are listed in Table 1.

Note that the excitation frequency is now denoted $\Omega$. In the case of primary resonance we will set $\Omega=\omega$ and search for solution under Fourier series with fundamental frequency $\omega$ (see Eq. (2)). In the case of subharmonic resonances we will set $\Omega=n \omega$ and still search for Fourier series with fundamental frequency $\omega=\Omega / n$. This approach provides a convenient way to decrease the fundamental frequency of the Fourier series and therefore expand harmonic content without any procedural modification.

Lastly, the selection parameters for the AHBM are set to $\rho_{f}=10^{-8}$ and $\rho_{b}=10^{-10}$ for the remainder of this example.

\subsubsection{Primary resonance}

A first simulation is carried out to obtain the primary system resonances. As explained in the previous section, the excitation frequency $\Omega$ is equal to the fundamental frequency $\omega$ in the development in Eq. (2). The maximum number of harmonics is set equal to $N_{h}=20$ for HBM and $N_{h}^{m}=20$ for AHBM. In this case the development in Eq. (2) includes both even and odd multiples of the fundamental frequency $\omega=\Omega$. The nonlinear algebraic system given by the HBM (or the AHBM) can then be solved by means of an arc-length continuation. Lastly, the excitation frequency is expressed as $\Omega=\omega_{2}(1+\sigma)$ with $\sigma \in[-0.05 ; 0.15]$.

The frequency response is depicted in Fig. 2 while the number of retained variables for each dof is depicted in Fig. 3(a,b). In order to clarify the results presentation (no overlapping on the plot due to turning point and thus multiple solutions for the same detuning value) the $x$-axis in Fig. 3(a, b) has been changed from $\sigma$ to "point number" corresponding to the position on the curve (Fig. 2). Although the number of variable have been divided by more than 4 , a good match still remains between solutions. The protocol maintains odd harmonics up to the fifth depending on the excitation frequency and, as expected, removes all even harmonics. In the end, 10 even harmonics and at least seven odd harmonics will have been removed by applying the selection procedure. The proximity of the two solutions is illustrated by depicting the following error estimators:

- the relative error between HBM and AHBM solutions:

$$
\epsilon_{\text {sol }}=\frac{\left\|\tilde{\boldsymbol{U}}_{H B M}-\tilde{\boldsymbol{U}}_{\text {AHBM }}\right\|}{\left\|\tilde{\boldsymbol{U}}_{H B M}\right\|}
$$

- relative error between strain energy:

$$
\epsilon_{\text {strain }}=\frac{\left|S_{\text {HBM }}-S_{\text {AHBM }}\right|}{\left|S_{\text {HBM }}\right|}
$$

with

$$
\left.S_{\text {Meth }}=\frac{1}{2} \frac{\omega}{2 \pi} \int_{0}^{2 \pi / \omega} T \boldsymbol{U}_{\text {Meth }}(t) \mathbf{K} \boldsymbol{U}_{\text {Meth }}(t) d t\right)
$$

Table 1

List of parameter used for the resolution of Eq. (20).

\begin{tabular}{lll}
\hline Param. & $i=1$ & $i=2$ \\
\hline$\omega_{i}$ & 3.8553 & 12.4927 \\
$c_{i}$ & 100 & 100 \\
$\alpha_{i, 1}$ & 8.27 & 30.8 \\
$\alpha_{i, 2}$ & 9.24 & 33.12 \\
$\alpha_{i, 3}$ & 33.12 & 34.41 \\
$\alpha_{i, 4}$ & 11.47 & 114.84 \\
\hline
\end{tabular}



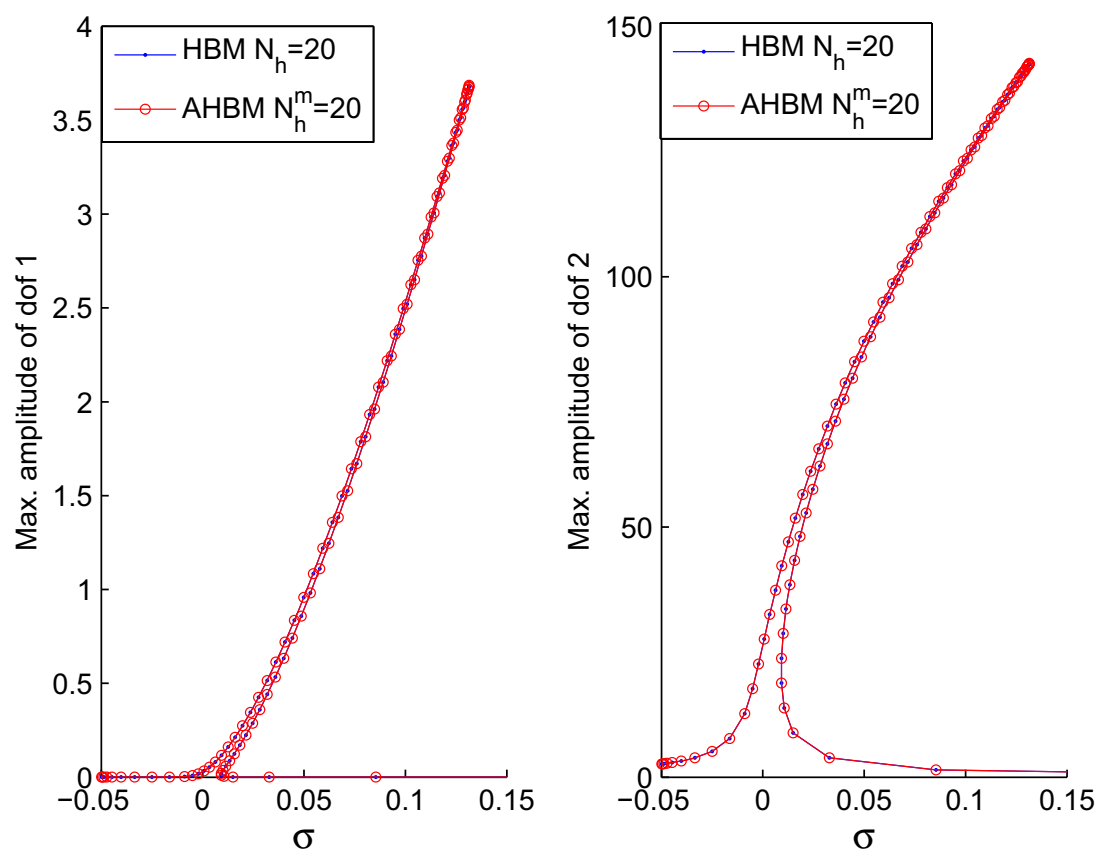

Fig. 2. Frequency response for the primary resonance of system in Eq. (20) (left: dof 1, right: dof 2).
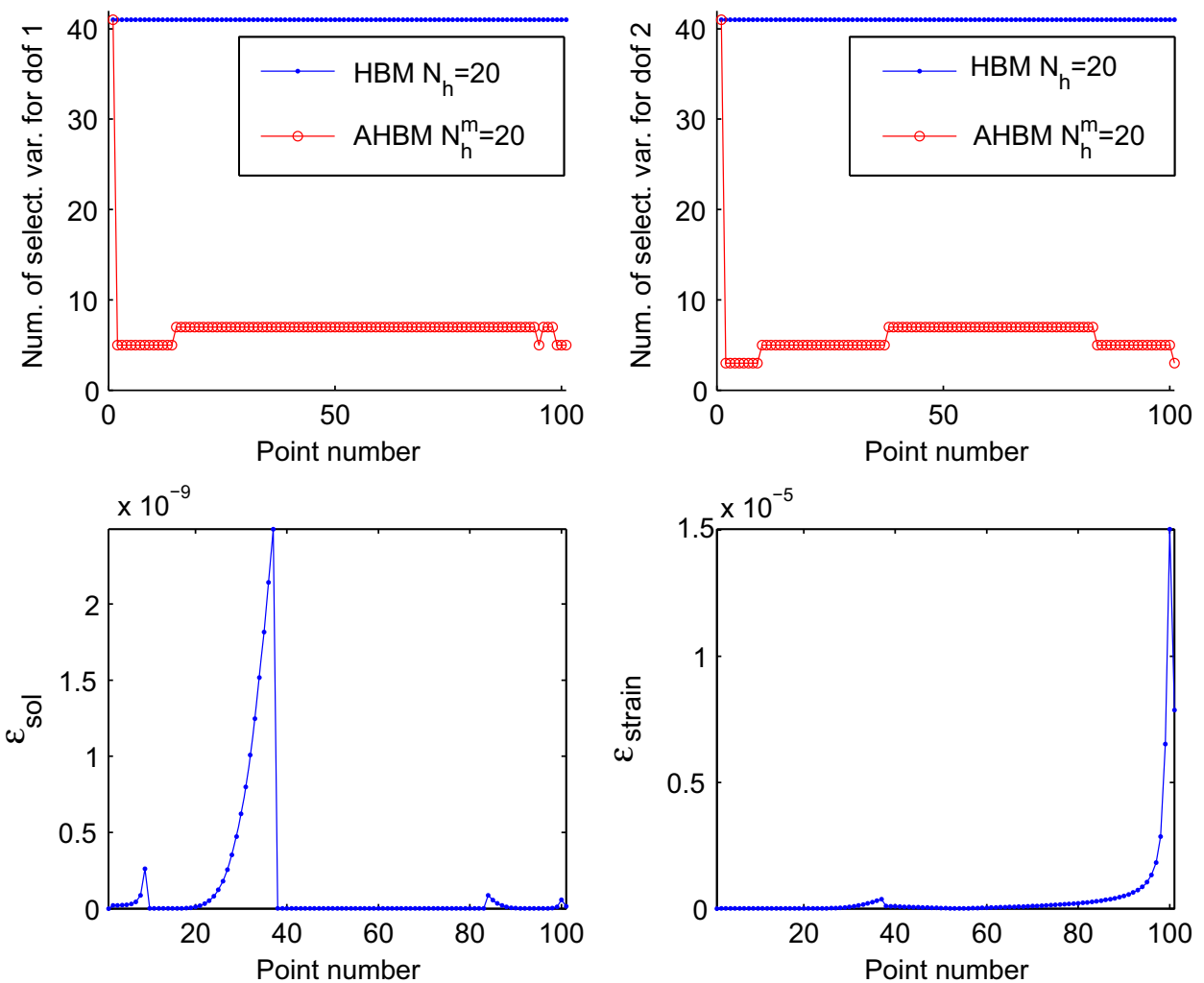

Fig. 3. Number of selected variable and error estimator for the primary resonance of system in Eq. (20). From top left to bottom right: (a) number of selected variable for dof 1, (b) number of selected variable for dof 2, (c) relative error on solution $\epsilon_{\text {sol }}$ and (d) relative error on (linear) strain energy $\epsilon_{\text {strain }}$. 
in Fig. 3(c,d), where it is visible that both error estimators $\epsilon_{\text {sol }}$ and $\epsilon_{\text {strain }}$ defined in Eqs. (22) and (23) lie, respectively, under $2.5 \times 10^{-7} \%$ and $2 \times 10^{-3} \%$.

\subsubsection{Secondary resonance}

Let us compare the two natural frequencies given in Table 1 ; it can be seen that $\omega_{2}=3 \omega_{1}(1+0.08)$, which leads to conclude that a 1:3 internal resonance can occur in this system. Consequently, the present section will involve searching for the system subharmonic resonance induced by this $1: 3$ internal resonance.

As explain in Section 4.2.1 the excitation frequency of Eq. (20) is set at $\Omega=n \omega$ with $n=9$ which therefore yields a fundamental frequency of development in Eq. (2) of $\omega=\Omega / 9$. The maximum number of harmonic is set to $N_{h}=27$ for the HBM and $N_{h}^{m}=27$ for the AHBM. In this case the development in Eq. (2) contains frequencies of the form $k \Omega / 9$ from $k=1$ to 27 , hence the maximum frequency contained in this development would be $3 \Omega$.

Let us note that the search for a subharmonic solution is not trivial since it does not bifurcate from the primary resonance response. We were led to run a polynomial homotopy algorithm [7] at $\Omega=\omega_{2}(1+0.05)$ in order to derive a starting point for the arc length continuation method.

Once the starting point has been computed, this simulation is carried out with both HBM and AHBM. Frequency responses are depicted in Fig. 4 while the number of selected variables for both dof is depicted in Fig. 5(a,b) (where, as before, the $x$-axis has been changed to point number). The two methods yield very similar results, whereas the number of selected variable in the AHBM is less than half the number of HBM variables. This finding has been confirmed in Fig. 5 (bottom left and bottom right), where the two error estimators $\epsilon_{\text {sol }}$ and $\epsilon_{\text {strain }}$ defined in Eqs. (22) and (23) lie, respectively, below $10^{-6 \%}$ and $3 \times 10^{-2} \%$.

The harmonic selection method automatically remove all harmonics of the form $2 k \Omega / 9$ as would be expected. Moreover, a selection takes place between harmonics of the form $(2 k+1) \Omega / 9$. To illustrate this point, the harmonic content of the two dof are depicted in Figs. 6 and 7 (where only harmonics of the form $(2 k+1) \Omega / 9$ have been depicted). As an example, for dof 1 (Fig. 6), it is observed that the $5 / 9 \Omega$ harmonic is never selected, the $21 / 9 \Omega=7 / 3 \Omega$ is always selected, and the $19 / 9 \Omega$ can be either selected or not depending on the point number. These two figures also indicate that the response corresponds to a subharmonic resonance induced by an internal resonance. It is in fact clearly visible that the dominant frequency for dof 1 is $1 / 3 \Omega$ while the dominant frequency for dof 2 is $\Omega$.

As a final step, results are compared with a direct integration of motion equations for an excitation frequency of $\Omega=w_{2}(1+0.0536)$ (which correspond to the point number 10 ). Results are plotted in Fig. 8, revealing a perfect match between solutions.

In conclusion, the harmonic selection procedure may be applied even in the presence of internal resonance phenomena. As a matter of fact, the selection procedure could prove quite useful in such cases. When solving these systems using HBM the fundamental frequency $\omega=\Omega / n$ can be set very small (i.e. with large $n$ ) leading to many (insignificant) terms in the
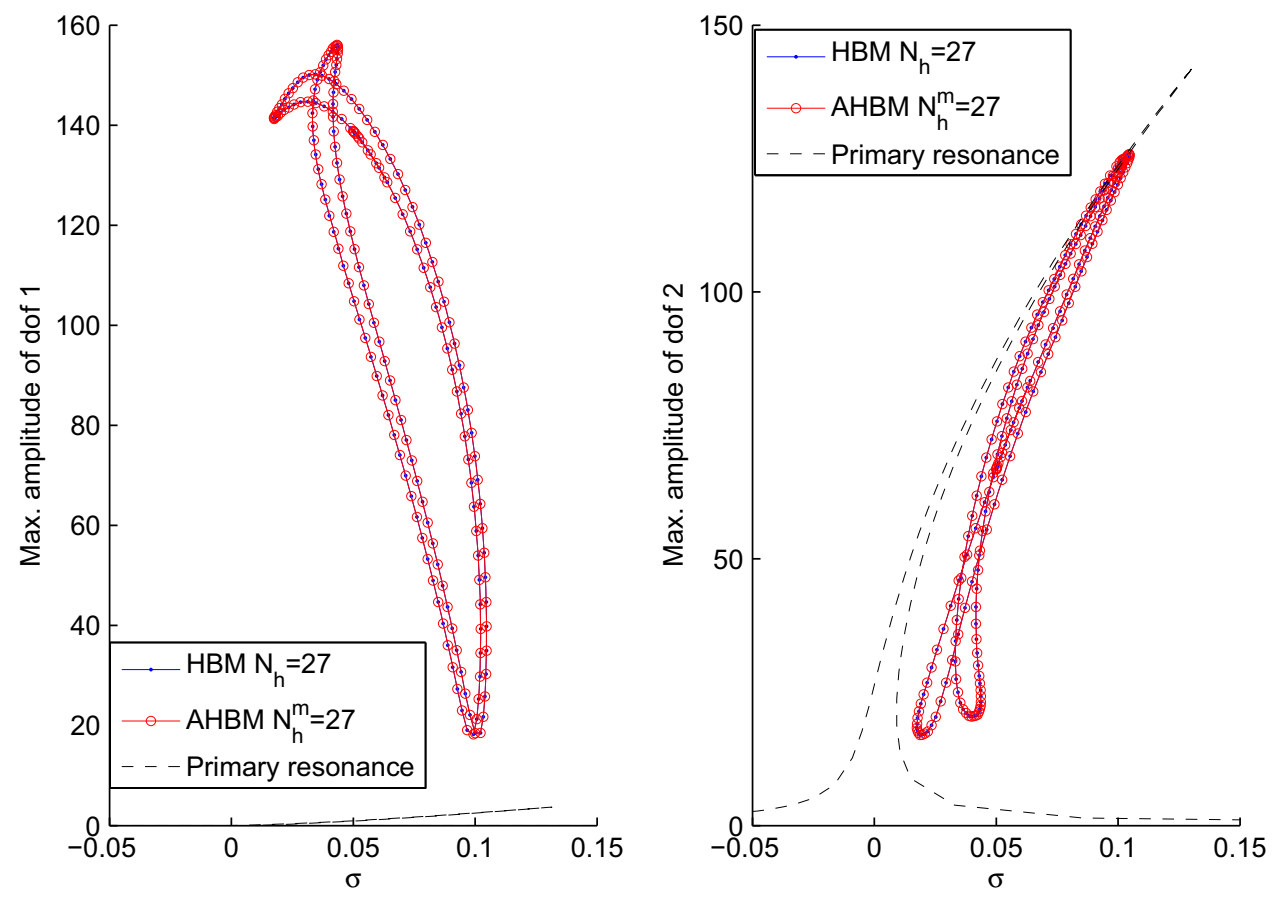

Fig. 4. Frequency response for the secondary resonance of system in Eq. (20) (left: dof 1, right: dof 2). 

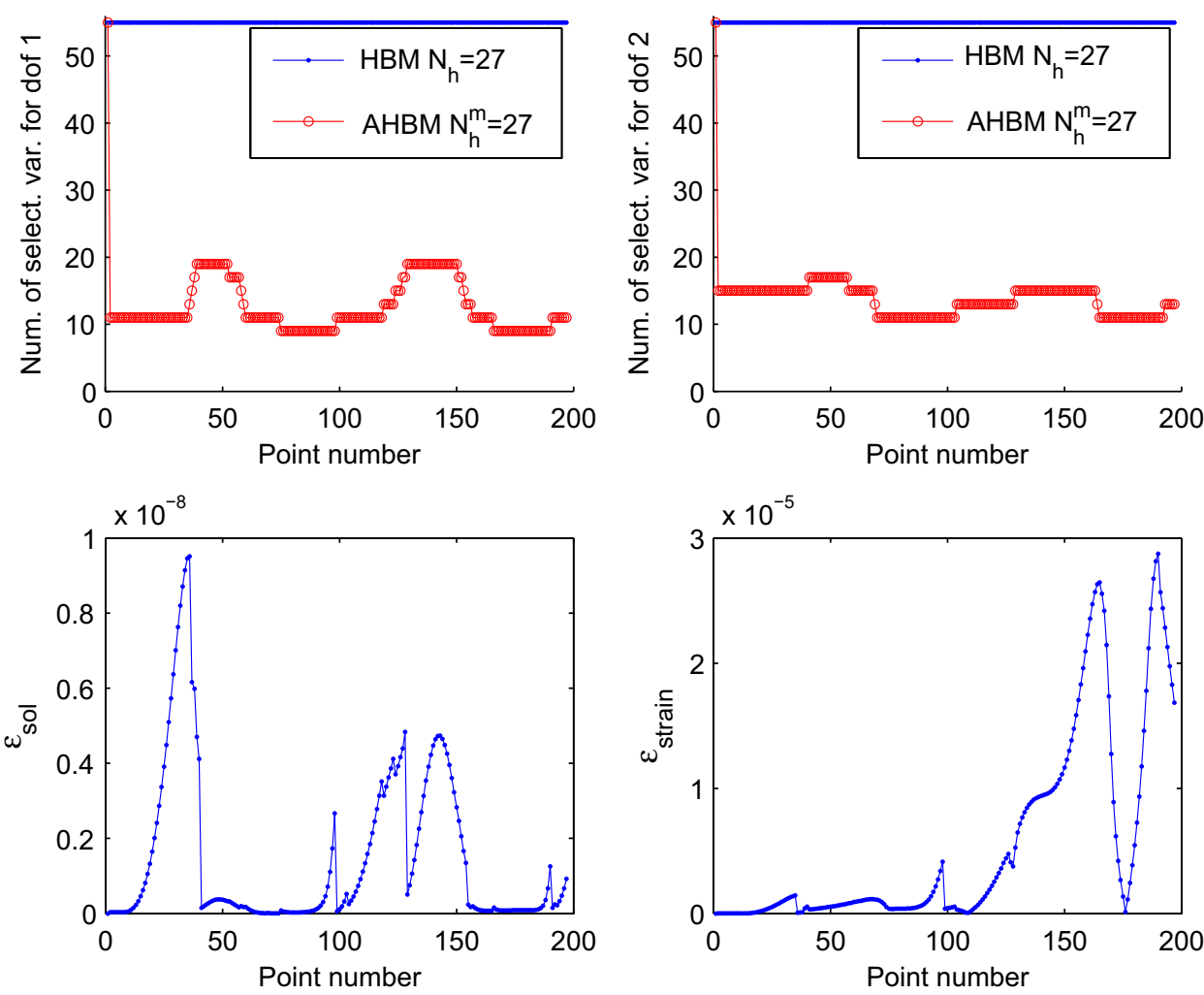

Fig. 5. Number of selected variable and error estimator for the secondary resonance of system in Eq. (20). From top left to bottom right: (a) number of selected variable for dof 1 , (b) number of selected variable for dof 2, (c) relative error on solution $\epsilon_{\text {sol }}$ and (d) relative error on (linear) strain energy $\epsilon_{\text {strain }}$.

Fourier development expressed in Eq. (2). The AHBM proposed herein is able to automatically suppress all useless terms, thus offering a smaller set of equation to be solved.

\subsection{Example 2: An industrial problem}

\subsubsection{System definition}

The system studied in this example consists of a blade and a disk sector in rotation bounded by a dovetail joint where friction can occur. The finite element meshes of the blade and the disk are shown in Fig. 9(a). There is two contact interfaces and the meshes are assumed to be compatible at each contact interface. No boundary conditions are applied on the blade (i.e. a free structure), and a zero displacement condition is imposed on the sector edges. Each structure is subjected to centrifugal forces. From this FE model, a Craig-Bampton reduced order model was built, which entailed retaining a set of $N_{\text {node }}$ nodes (for the blade and the disk) at each contact interfaces, plus another node at the blade tip trailing edge (which acts as a control node). In addition $N_{\text {mode }}$ dynamic modes with fixed interface were considered in the Craig-Bampton reduction for each structure. At the interfaces, the unilateral contact law and the Coulomb friction law are used in this example. Further details on this model are available in $[5,6]$.

The resulting equation of motion for each structure $s=1,2$ is given by

$$
\mathbf{M}^{s} \ddot{U}^{s}+\mathbf{C}^{s} \dot{U}^{s}+\mathbf{K}^{s} U^{s}+\boldsymbol{F}_{n l}^{s}=\boldsymbol{F}_{e x}^{s}
$$

where $\mathbf{M}^{s}$ (resp., $\mathbf{C}^{s}, \mathbf{K}^{s}$ ) represent the mass (resp., the damping and the stiffness) matrix, $\boldsymbol{F}_{n l}^{s}$ represents the nonlinear forces and $\boldsymbol{F}_{e x}$ the excitation force.

Since the nonlinear forces are only acting at the contact interface, the motion equations can be split into the following form:

$$
\left[\begin{array}{ll}
\mathbf{M}_{c c}^{s} & \mathbf{M}_{c a}^{s} \\
\mathbf{M}_{a c}^{s} & \mathbf{M}_{a a}^{s}
\end{array}\right]\left[\begin{array}{c}
\ddot{U}_{c}^{s} \\
\ddot{U}_{a}^{s}
\end{array}\right]+\left[\begin{array}{cc}
\mathbf{C}_{c c}^{s} & \mathbf{C}_{c a}^{s} \\
\mathbf{C}_{a c}^{s} & \mathbf{C}_{a a}^{s}
\end{array}\right]\left[\begin{array}{c}
\dot{U}_{c}^{s} \\
\dot{U}_{a}^{s}
\end{array}\right]+\left[\begin{array}{cc}
\mathbf{K}_{c c}^{s} & \mathbf{K}_{c a}^{s} \\
\mathbf{K}_{a c}^{s} & \mathbf{K}_{a a}^{s}
\end{array}\right]\left[\begin{array}{c}
U_{c}^{s} \\
U_{a}^{s}
\end{array}\right]+\left[\begin{array}{c}
\boldsymbol{F}_{c}^{s} \\
\mathbf{0}
\end{array}\right]=\left[\begin{array}{c}
\boldsymbol{F}_{e x, c}^{s} \\
\boldsymbol{F}_{e x, a}^{s}
\end{array}\right]
$$

where subscript $c$ (resp. $a$ ) denotes for the contact nodes (resp. the linear nodes). Matrices are projected in a local frame such that the vector of relative displacements at the contact interface is expressed as $\boldsymbol{U}_{r}=\boldsymbol{U}_{c}^{1}-\boldsymbol{U}_{c}^{2}$ and such that the action-reaction principle reads $\boldsymbol{F}_{c}^{1}=-\boldsymbol{F}_{c}^{2}$. 

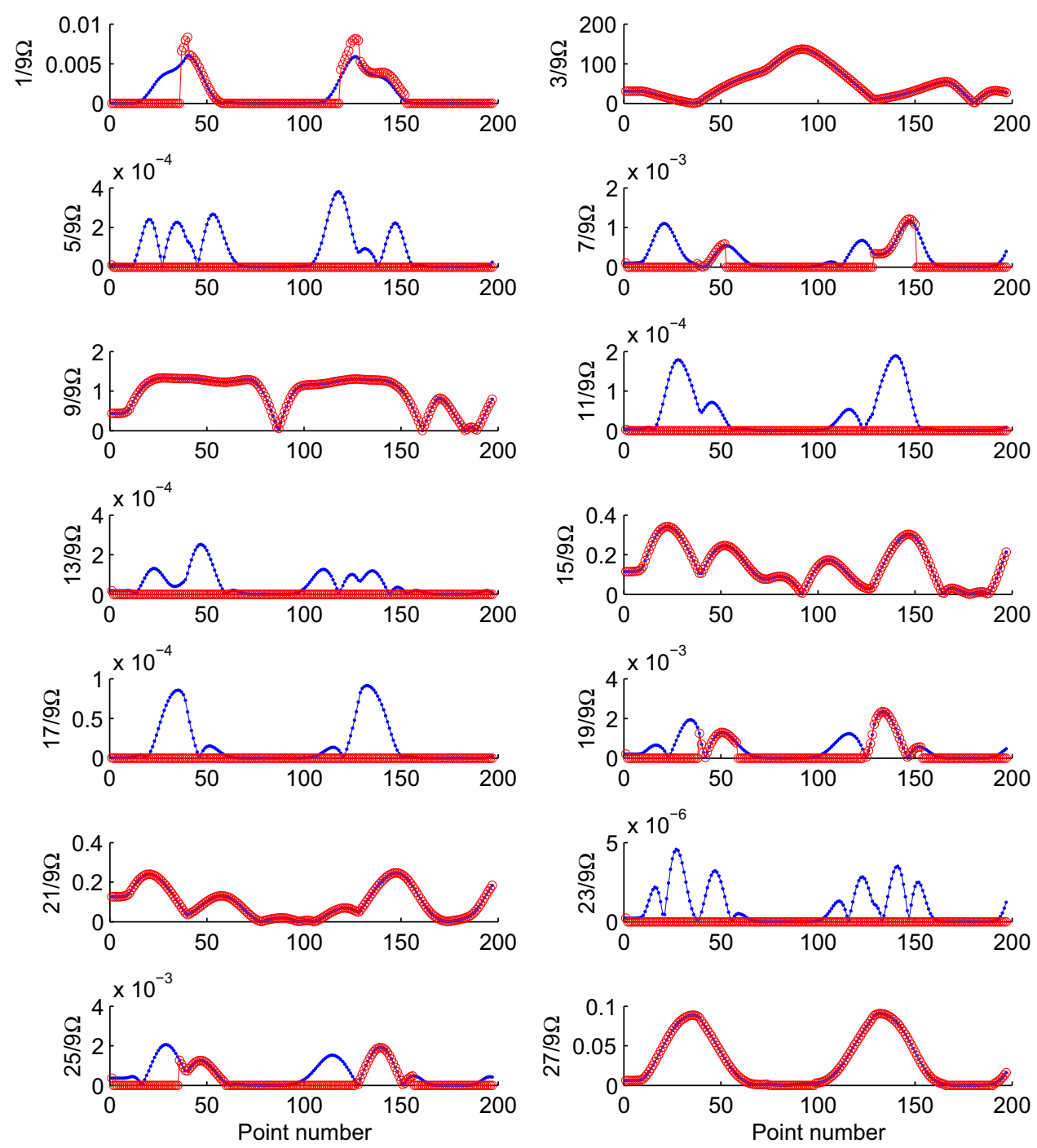

Fig. 6. Amplitude of odd harmonics of dof 1 in the case of a secondary resonance $(-\cdot-$ : HBM, $-\circ-:$ AHBM).

After applying the HBM projection (see Appendix A), the following motion equation is obtained in the frequency domain for each structure s:

$$
\left[\begin{array}{cc}
\boldsymbol{\Lambda}_{c c}^{s} & \boldsymbol{\Lambda}_{c a}^{s} \\
\boldsymbol{\Lambda}_{a c}^{s} & \boldsymbol{\Lambda}_{a a}^{s}
\end{array}\right]\left[\begin{array}{c}
\tilde{U}_{c}^{s} \\
\tilde{U}_{a}^{s}
\end{array}\right]+\left[\begin{array}{c}
\tilde{F}_{c}^{s} \\
\tilde{0}
\end{array}\right]=\left[\begin{array}{c}
\tilde{F}_{e x, c}^{s} \\
\tilde{F}_{e x, a}^{s}
\end{array}\right]
$$

\subsubsection{Determination of the contact forces}

The friction forces $\tilde{F}_{c}^{s}$ are evaluated with a dynamic Lagrangian frequency-time (DLFT) approach [17]. This technique uses augmented Lagrangians which allow for calculation without any softening of the non-smooth contact and frictional laws. A time marching procedure in the time domain is also required to fit the contact constraint. The main advantage of this method is that, at convergence, results are not dependent on any penalty coefficients. In most research focusing on DLFT [5,17], motion equations are condensed on the nonlinear dof and on the relative dof. Since in this example one of the structures is free we would prefer to avoid condensing our model in order to both eliminate any potential numerical issues (i.e. singularity of the stiffness matrix for the blade), and witness the effect of the selection procedure on all dofs. In the frequency domain the contact forces are expressed as a penalization of the motion equations; however, since no condensation has been applied, the definition of contact force differs slightly from that in [17]. We have defined contact forces herein as follows:

$$
\tilde{\boldsymbol{\lambda}}=\tilde{\boldsymbol{F}}_{c}^{1}=-\tilde{\boldsymbol{F}}_{c}^{2}=\frac{1}{2}\left(\tilde{\boldsymbol{F}}_{e x, c}^{1}-\boldsymbol{\Lambda}_{c c}^{1} \tilde{\boldsymbol{U}}_{c}^{1}-\boldsymbol{\Lambda}_{c a}^{1} \tilde{\boldsymbol{U}}_{a}^{1}-\left(\tilde{\boldsymbol{F}}_{e x, c}^{2}-\boldsymbol{\Lambda}_{c c}^{2} \tilde{\boldsymbol{U}}_{c}^{2}-\boldsymbol{\Lambda}_{c a}^{2} \tilde{\boldsymbol{U}}_{a}^{2}\right)\right)+\epsilon\left(\tilde{\boldsymbol{U}}_{c}^{1}-\tilde{\boldsymbol{U}}_{c}^{2}-\tilde{\boldsymbol{X}}_{r}\right)
$$



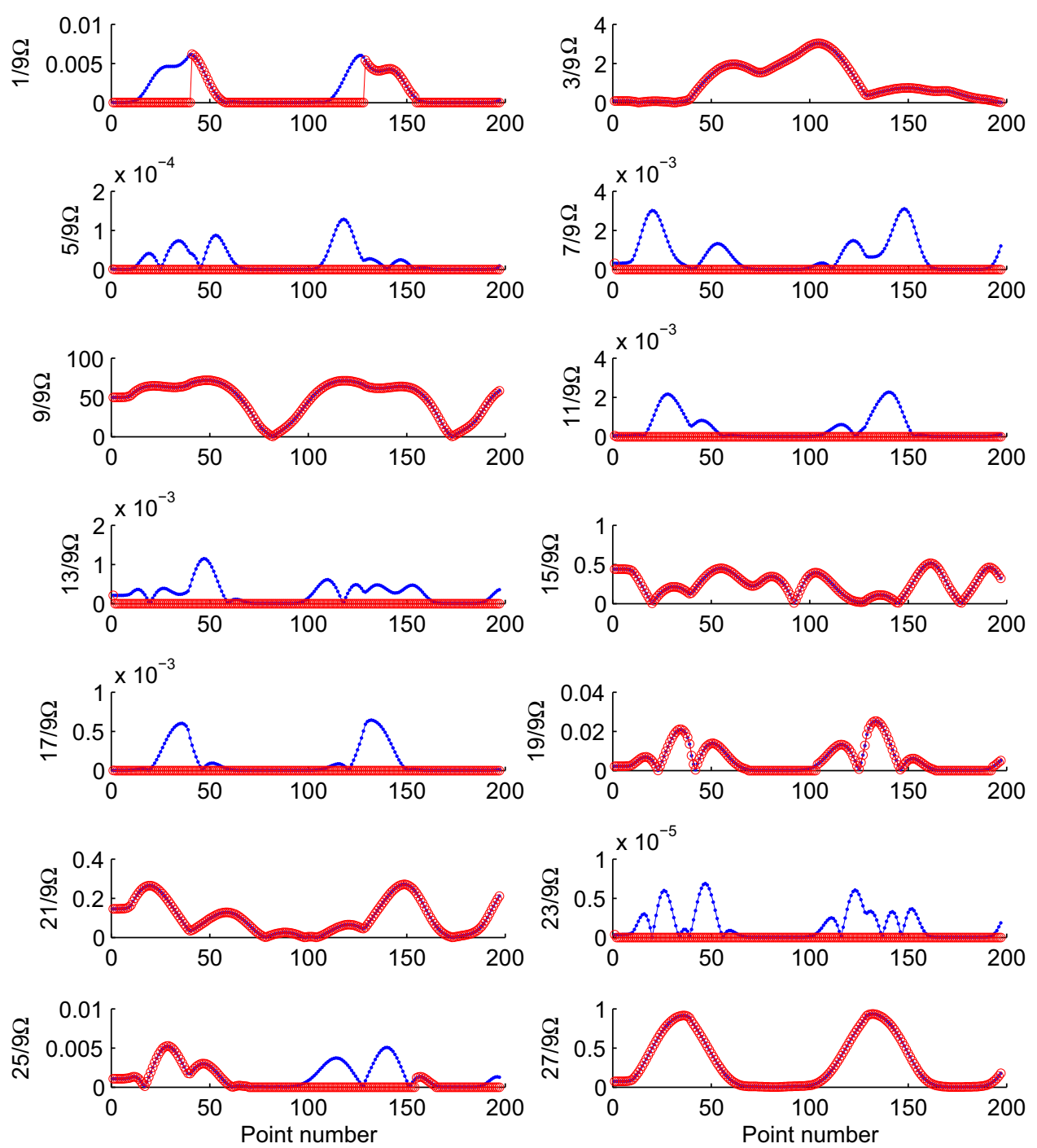

Fig. 7. Amplitude of odd harmonics of dof 2 in the case of a secondary resonance $\left(-\cdot-:\right.$ HBM, $-\mathrm{O}_{-}:$AHBM).

where $\epsilon$ is the (pseudo) penalty coefficient and $\tilde{\boldsymbol{X}}_{r}$ is a new variable of relative displacement computed in the time domain so as to ensure compliance with both the contact and friction laws. In theory the penalty coefficient $\epsilon$ can be chosen arbitrarily since, at convergence, results do not depend on $\epsilon$. However Charleux proposed to take $\epsilon$ in the order of magnitude of the spectral radius of the stiffness matrix $\mathbf{K}$ [5]. In this example we took $\epsilon=10^{4}$.

The couple $\left(\tilde{\boldsymbol{\lambda}}, \tilde{\boldsymbol{X}}_{r}\right)$ is determined through an AFT procedure as detailed in [17].

The contact forces will be divided as follows:

$$
\tilde{\lambda}=\tilde{\lambda}_{\tilde{\boldsymbol{u}}}-\tilde{\boldsymbol{\lambda}}_{\tilde{\boldsymbol{x}}}
$$

with

$$
\begin{aligned}
& \tilde{\boldsymbol{\lambda}}_{\tilde{\boldsymbol{u}}}=\frac{1}{2}\left(\tilde{\boldsymbol{F}}_{e x, c}^{1}-\boldsymbol{\Lambda}_{c c}^{1} \tilde{\boldsymbol{U}}_{c}^{1}-\boldsymbol{\Lambda}_{c a}^{1} \tilde{\boldsymbol{U}}_{a}^{1}-\left(\tilde{\boldsymbol{F}}_{e x, c}^{2}-\boldsymbol{\Lambda}_{c c}^{2} \tilde{\boldsymbol{U}}_{c}^{2}-\boldsymbol{\Lambda}_{c a}^{2} \tilde{\boldsymbol{U}}_{a}^{2}\right)\right)+\epsilon\left(\tilde{\boldsymbol{U}}_{c}^{1}-\tilde{\boldsymbol{U}}_{c}^{2}\right) \\
& \tilde{\lambda}_{\tilde{\boldsymbol{x}}}=\epsilon \tilde{\boldsymbol{X}}_{r}
\end{aligned}
$$

We will now briefly recall the main steps involved in calculating $\tilde{\lambda}$ :

- From a given vector $\tilde{\boldsymbol{U}}=\left[\tilde{\boldsymbol{U}}^{1} ; \tilde{\boldsymbol{U}}^{2}\right]$, compute the vector $\tilde{\boldsymbol{\lambda}}_{\tilde{\boldsymbol{u}}}$ in the frequency domain along with its counterpart $\lambda_{\tilde{\boldsymbol{u}}}$ in the time domain.

- For each time step $n$, a prediction $\lambda_{\text {pre }}^{n}$ of the contact force in the time domain is computed under the assumption that the nodes had not moved from their position at time step $n-1$. 

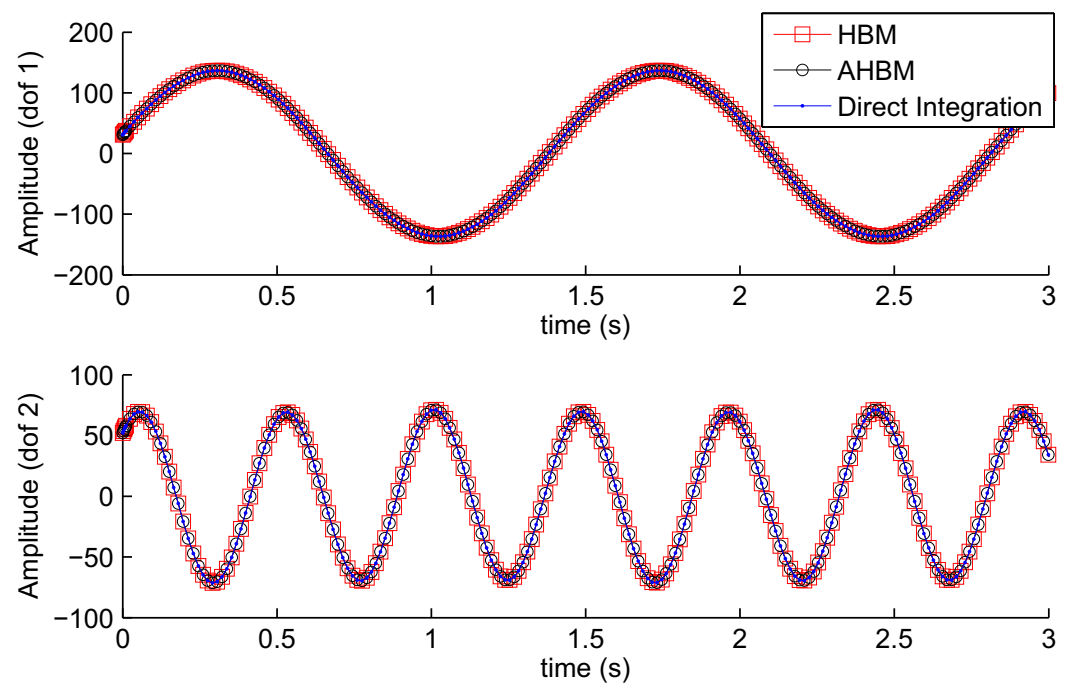

Fig. 8. Comparison between direct integration, HBM and AHBM for the case of secondary resonance of system in Eq. (20) for $\Omega=w_{2}(1+0.0536)$ (top: amplitude of dof 1, bottom: amplitude of dof 2).
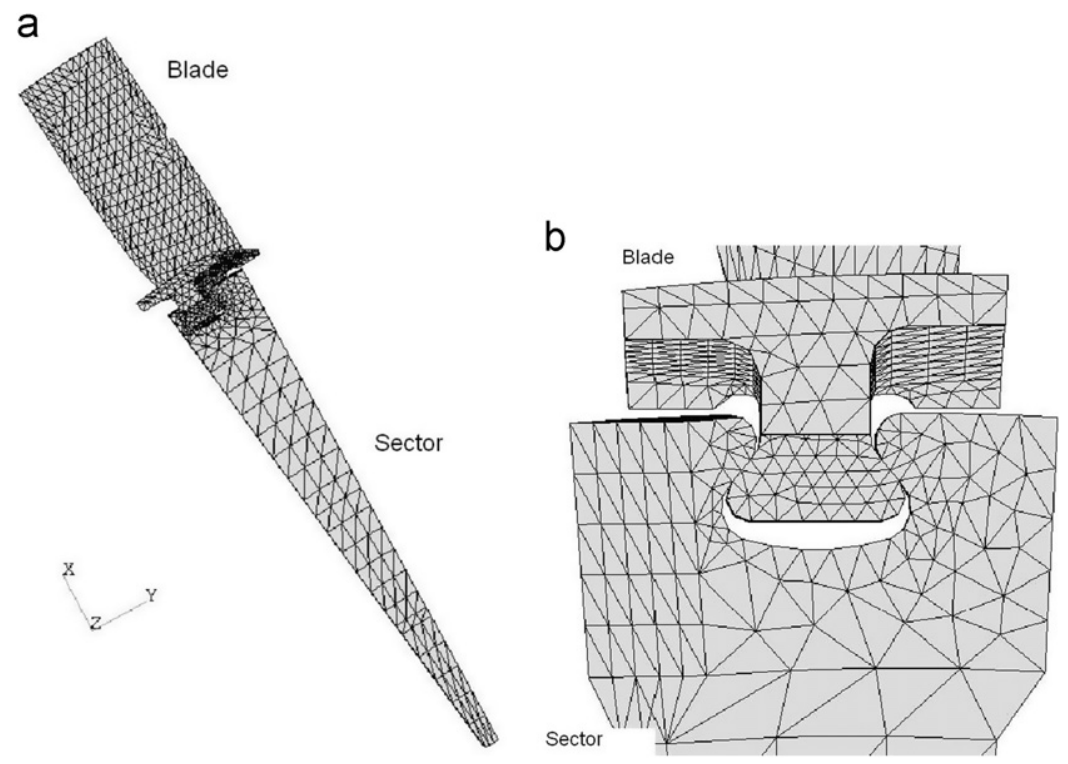

Fig. 9. Finite element model of the system considered in example 2: (a) blade and sector FEM; (b) detailed view of the dovetail joint.

- $\lambda_{\tilde{\boldsymbol{x}}}^{n}$ is computed in the time domain in order to satisfy the contact and friction states (separation, stick, slip) given by the prediction $\lambda_{\text {pre}}^{n}$.

- $\lambda$ is computed using the relation $\lambda^{n}=\lambda_{\tilde{u}}^{n}-\lambda_{\tilde{\boldsymbol{x}}}^{n}$, and $\tilde{\lambda}$ is given by a FFT of $\lambda$.

Additional details about can be found in [17], which provide clear explanations of each step of the contact forces computation procedure.

\subsubsection{Simulations}

The simulations have been carried out with a reduced model in which $2 \times(3 \times 8)=48$ nodes have been retained on the contact interface. The number of dynamic modes retains in the Craig-Bampton reduction equals 11 for the blade and the sector. We ultimately produced a model with $n_{1}=159$ dofs for the blade $(=[(48$ nonlinear nodes +1 control node $) \times 3$ dof per node +11 modes $])$ and $n_{2}=156$ dofs for the sector $(=[144 \times 3+11])$.

For this simulation, a harmonic force $f$ assuming the form $f(t)=A \cos (\omega t)$ has been applied on the normal component of the control node. The force amplitude is set at $A=0.2$. Simulation takes place around the first mode of the system, and the 


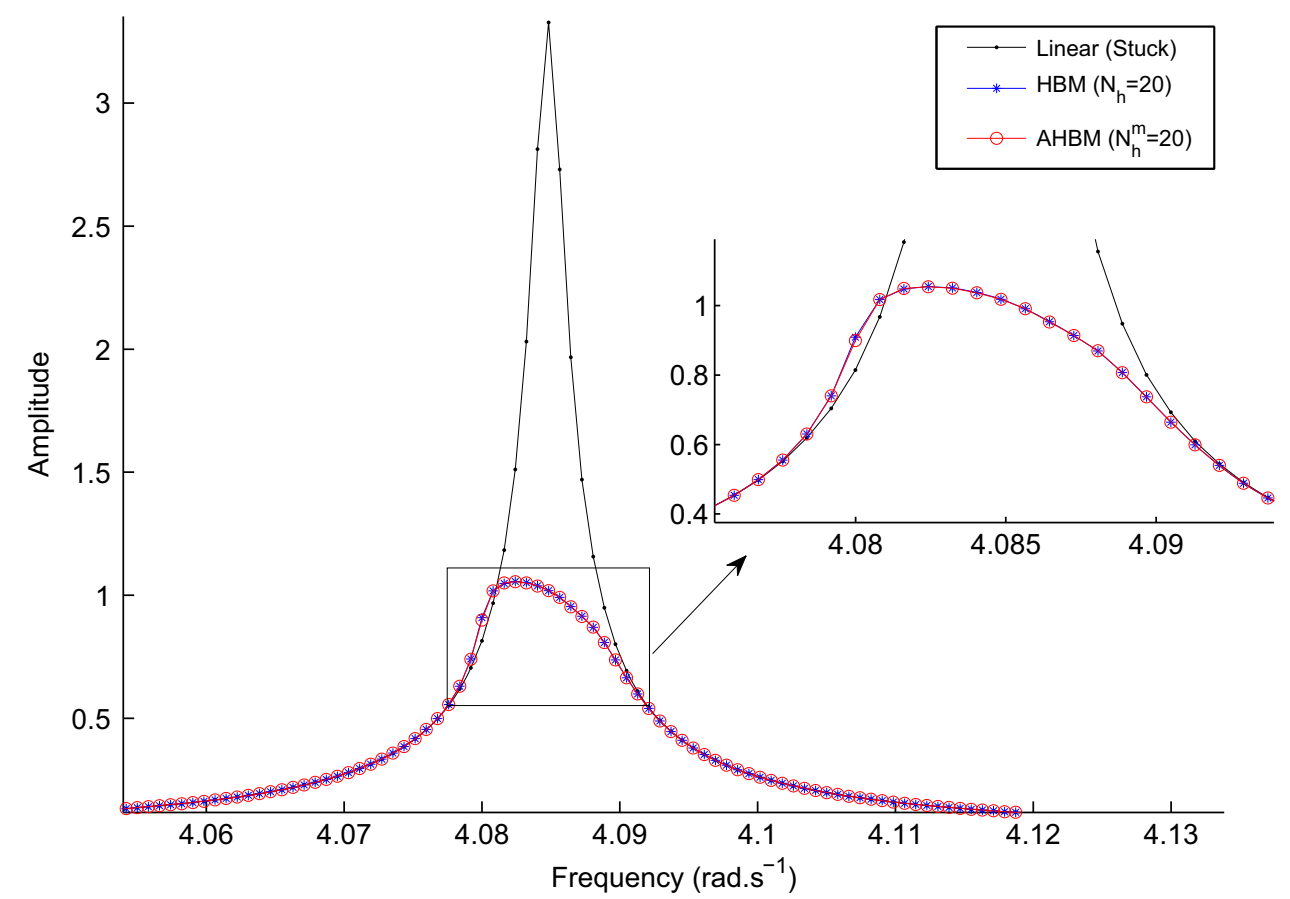

Fig. 10. Amplitude response of the control node in the normal direction: comparison between HBM (*) and AHBM ( $O)$.

problem is solved with HBM with $N_{h}=20$ harmonics (leading to a problem with 12915 unknowns) and AHBM with $N_{h}^{m}=20$ maximum harmonics. Selection parameters have been set at $\rho_{b}=10^{-10}$ and $\rho_{f}=10^{-8}$. The Jacobian matrix used to compute the prediction (Eq. (16)) is computed semi-analytically as indicated in [17]. Note that in this simulation all harmonics (continuous component, odd and even harmonics) have been retained. From a preliminary computation with only one harmonic, we were able to observe that the continuous component of the solution is greater than its harmonic component; therefore, the parameters $\epsilon_{k}^{d}$ of Eq. (17) have been computed without the continuous component in order to avoid rounding off errors, as discussed in Section 3.3.

Fig. 10 shows the amplitude response of the control dof in the normal direction, no differences can be observed between HBM and AHBM results. Fig. 11 displays the various kinds of error. This illustration confirms that HBM and AHBM solutions are very close to one another since the relative error between HBM and AHBM solutions (Eq. (22)) is less than $42 \times 10^{-4} \%$ for the blade (Fig. 11b) and the sector (Fig. 11c). The relative difference between strain energy (Eq. (23)) is also small, lying below $1.5 \%$ for the blade (Fig. 11d) and below $0.04 \%$ for the sector (Fig. 11e). Lastly, Fig. 11a reveals the error on motion equations defined by the following:

$$
\epsilon_{e q}=\int_{0}^{2 \pi / \omega}\left\|\mathbf{M} \ddot{\boldsymbol{X}}+\mathbf{C} \dot{\boldsymbol{X}}+\mathbf{K} \boldsymbol{X}+\boldsymbol{F}_{f r}(\dot{\boldsymbol{X}})-\boldsymbol{F}_{e x}\right\| d t
$$

once again confirming that the HBM and AHBM solutions are very close to each other.

The number of selected Fourier coefficients during the computation is depicted in Fig. $12 \mathrm{~d}$. It can be seen that the number of variables is significantly reduced compared to HBM. Solutions lying out of the resonance zone are computed with only $7.3 \%$ of the total number of variables ( 945 Fourier coefficients for the AHBM vs. 12915 Fourier coefficients for the HBM) whereas inside the resonance zone the number of selected Fourier coefficients increases until reaching a maximum corresponding to 93\% of the total number of variables (12 000 Fourier coefficients for the AHBM against 12915 Fourier coefficients for the HBM). Figs. 13-15 present the matrices of selected Fourier coefficients for the blade at three different frequencies. These figures indicate that solutions lying out of the resonance zone are computed with just the continuous component and the first harmonic (Fig. 13), then when approaching the resonance, some Fourier coefficients are added (Fig. 14), and finally in the resonance zone nearly all Fourier coefficients of all dof are selected (Fig. 15). The selection procedure leads to a total time savings on the order of $50 \%$ (Fig. 12).

\section{Conclusion}

This paper has introduced a new adaptive harmonic balance method and applied it to two examples. The harmonic selection technique relies upon both a tangent predictor computation and a spectral energy evaluation within the predicted vector. Unlike most existing adaptive harmonic balance methods, this new method defines a number of selected 

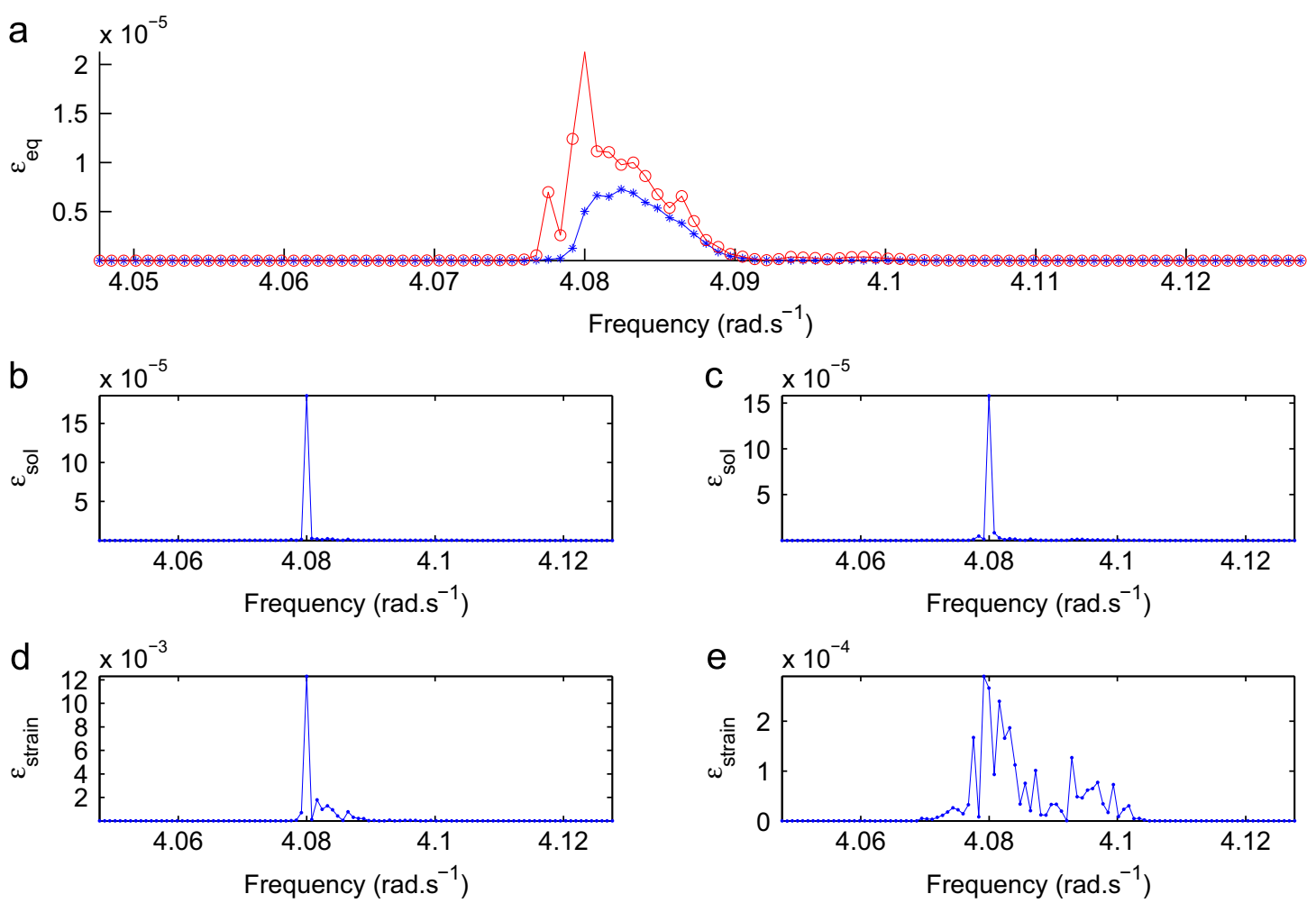

Fig. 11. Comparison of various errors between $\operatorname{HBM}(*)$ and AHBM ( $\bigcirc)$. From top left to bottom right: (a) error on motion equation $\epsilon_{e q}$; (b, c) relative error between solution $\epsilon_{\text {sol }}$ (for the blade (b), for the disc (c)); (d, e) relative error between strain energy $\epsilon_{\text {strain }}$ (for the blade (d), for the disc (e)).

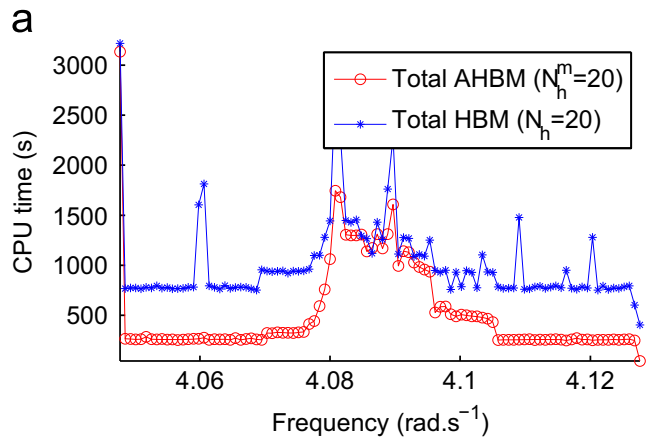

C

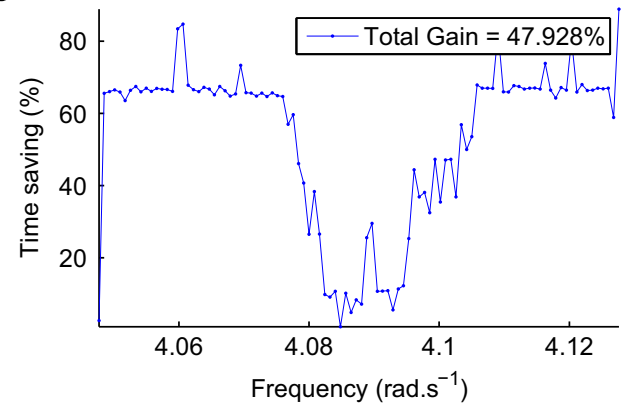

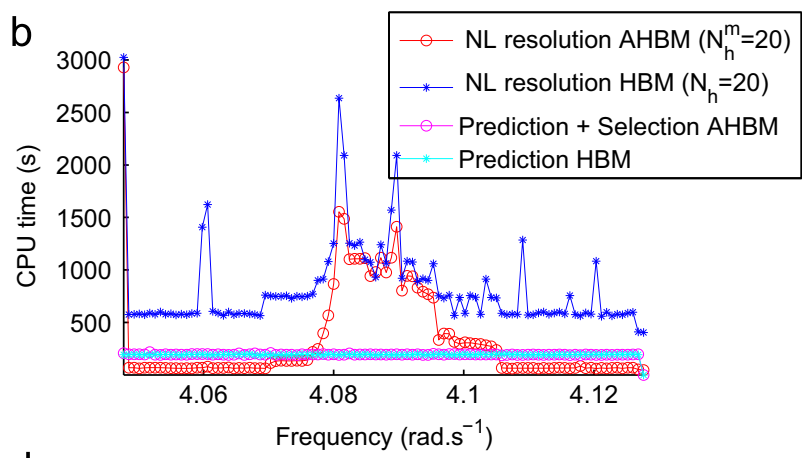

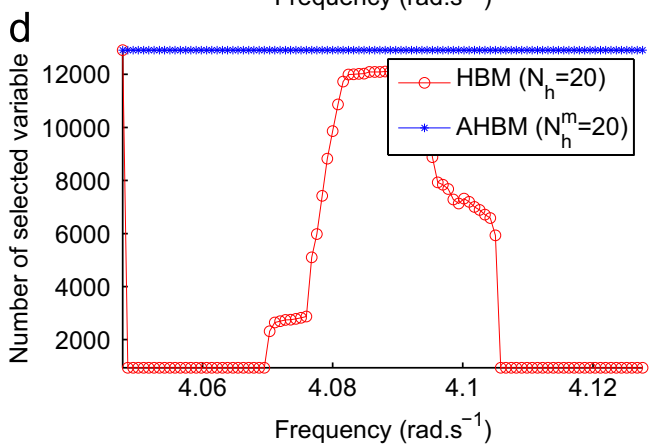

Fig. 12. Comparison of resolution time between HBM (*) and AHBM ( $\odot$ ). From top left to bottom right: (a) total resolution time; (b) detailed resolution time; (c) time savings; (d) number of selected variables. 


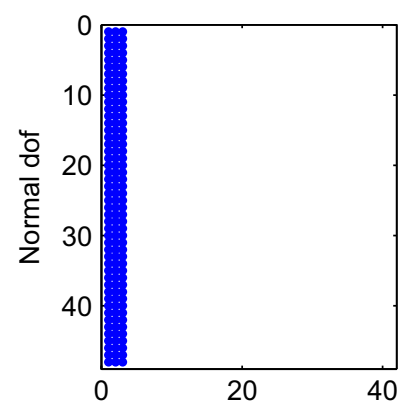

Fourier coefficient number

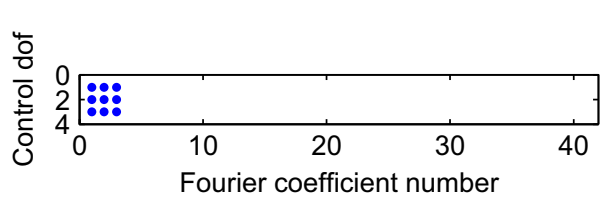

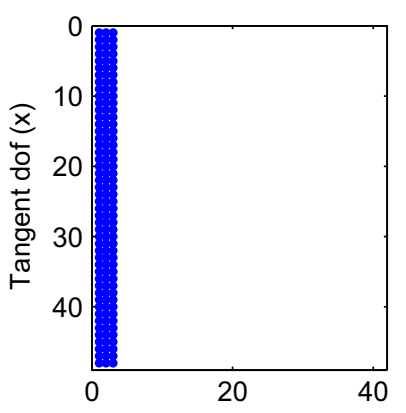

Fourier coefficient number

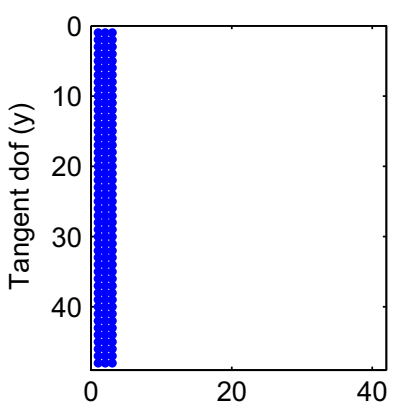

Fourier coefficient number

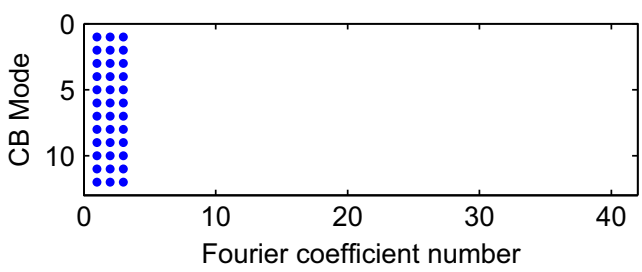

Fig. 13. Matrices of selected Fourier coefficients for the blade at $\omega=4.049$ rad s $^{-1}$. From top left to bottom right: normal dof, tangent dof ( $x$ direction), tangent dof ( $y$ direction), control node, CB modes.

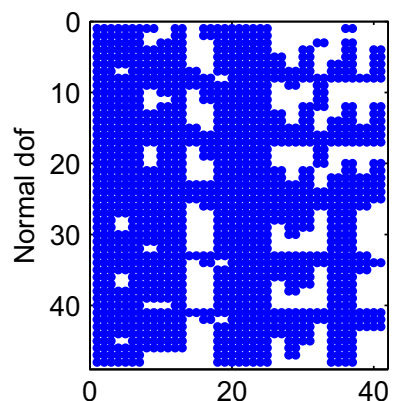

Fourier coefficient number

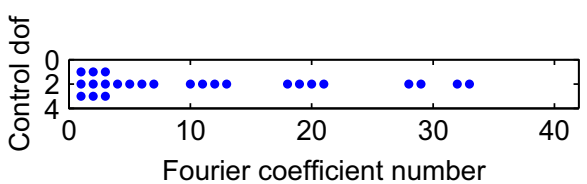

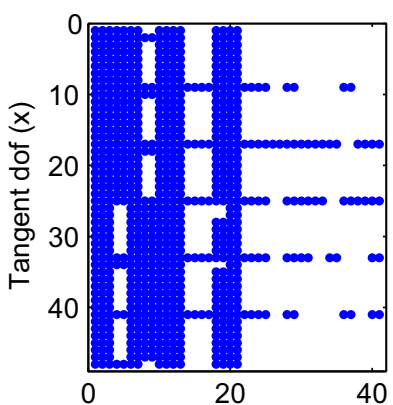

Fourier coefficient number

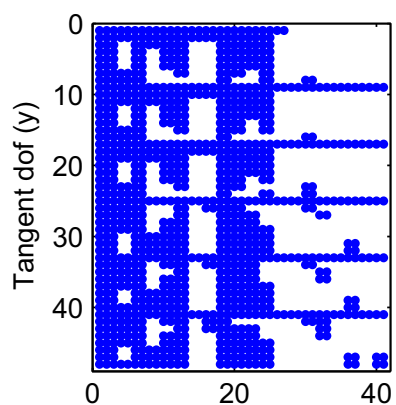

Fourier coefficient number

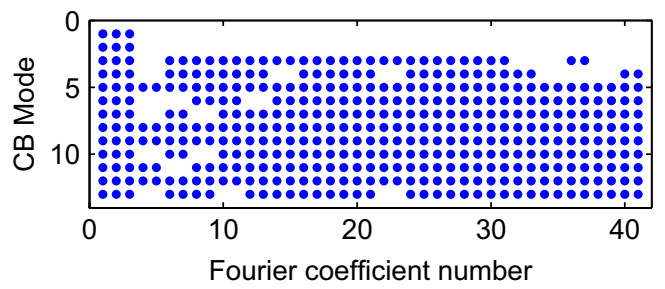

Fig. 14. Matrices of selected Fourier coefficients for the blade at $\omega=4.078 \mathrm{rad} \mathrm{s}^{-1}$. From top left to bottom right: normal dof, tangent dof ( $x$ direction), tangent dof ( $y$ direction) and control node and CB modes.

Fourier coefficients for each dof. Moreover, the addition or removal of variables does not require an $a$ priori increment on the number of harmonics: the selection algorithm on its own is able to determine which Fourier coefficient should be added or removed. The efficiency of this new AHBM has been demonstrated in various simulations conducted within the scope of this paper. Three examples have been considered, a small and simple system with smooth nonlinearity, a small system with cubic nonlinearity featuring internal resonance and an industrial problem with non-smooth nonlinearity. In all examples, when systems are excited in a quasi-linear zone (i.e. away from the resonance), solutions can be computed with a small number of Fourier coefficients; moreover, when systems are excited in the resonance zone, the number of retained Fourier coefficients varies with both dof and frequency. This procedure allows for time savings on the order of $50 \%$ compared with a classical HBM calculation and without deteriorating the quality of solutions. This method should allow handling large problems of great complexity, and especially problems presenting geometric nonlinearities or friction damping. 


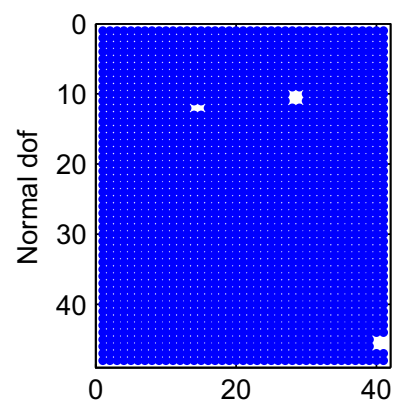

Fourier coefficient number

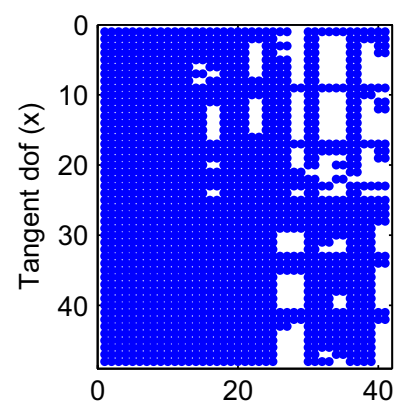

Fourier coefficient number

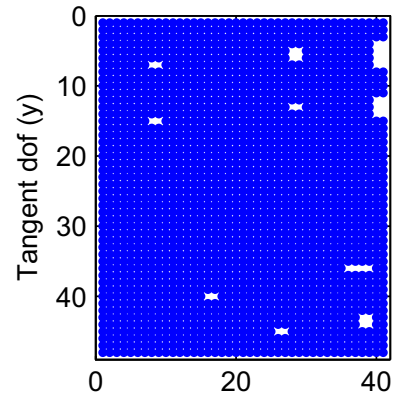

Fourier coefficient number
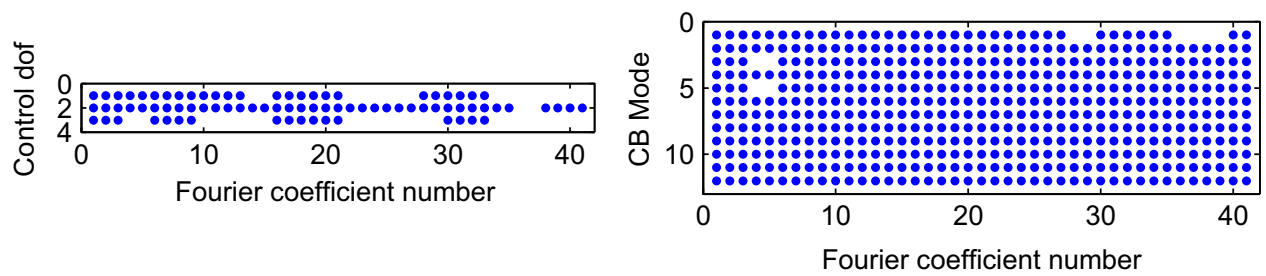

Fig. 15. Matrices of selected Fourier coefficients for the blade at $\omega=4.089 \mathrm{rad} \mathrm{s}^{-1}$. From top left to bottom right: normal dof, tangent dof $(x$ direction), tangent dof ( $y$ direction), control node and CB modes.

\section{Acknowledgments}

The authors would like to thank the Snecma Company for its technical and financial support. This research has been performed within the scope of the MAIA mechanical research and technology program sponsored by CNRS, ONERA and SAFRAN Group.

\section{Appendix A}

This appendix will review the harmonic balance method equations. Let us start by considering an $n$-dof nonlinear system whose motion equations are given as follows:

$$
\mathbf{M U}+\mathbf{C U}+\mathbf{K U}+\boldsymbol{F}_{n l}(\boldsymbol{U}, \dot{U})=\boldsymbol{F}_{e x}
$$

Periodic solutions $\boldsymbol{U}(t)$ of Eq. (A.1) are then sought according to the following form:

$$
\boldsymbol{U}(t)=\boldsymbol{U}_{0}+\sum_{k=1}^{N_{h}} \boldsymbol{U}_{k}^{c} \cos (k \omega t)+\boldsymbol{U}_{k}^{s} \sin (k \omega t)
$$

Next, by substituting Eq. (A.2) in Eq. (A.1) and projecting the expression obtained onto the $(1,[\cos (k \omega t), \sin (k \omega t)])$ basis using the following scalar product:

$$
\langle f, g\rangle=\int_{0}^{2 \pi / \omega} f(t) g(t) d t
$$

a set of $n\left(2 N_{h}+1\right)$ nonlinear algebraic equations can be derived as given by

$$
\boldsymbol{\Lambda}(\omega) \tilde{\boldsymbol{U}}+\tilde{\boldsymbol{F}}_{n l}=\tilde{\boldsymbol{F}}_{e x}
$$

with $\mathbf{\Lambda}(\omega)$ being the dynamic stiffness matrix, expressed as

$$
\begin{aligned}
& \mathbf{\Lambda}(\omega)=\operatorname{diag}\left(\mathbf{K}, \Lambda_{k}(\omega)_{1 \leq k \leq N_{h}}\right) \\
& \Lambda_{k}(\omega)=\left[\begin{array}{cc}
\mathbf{K}-(k \omega)^{2} \mathbf{M} & k \omega \mathbf{C} \\
-k \omega \mathbf{C} & \mathbf{K}-(k \omega)^{2} \mathbf{M}
\end{array}\right]
\end{aligned}
$$

$\tilde{\boldsymbol{U}}$ is the vector of unknowns, $\tilde{\boldsymbol{F}}_{n l}$ is the vector of nonlinear forces and $\tilde{\boldsymbol{F}}_{e x}$ is the vector of external forces. All these vector marked with a lie in the frequency domain and assume the following form for a given vector $\tilde{\boldsymbol{X}}$ :

$$
\tilde{\boldsymbol{X}}=\left[\boldsymbol{X}_{0}, \boldsymbol{X}_{1}^{c}, \boldsymbol{X}_{1}^{s}, \ldots, \boldsymbol{X}_{N_{h}}^{c}, \boldsymbol{X}_{N_{h}}^{s}\right]^{T}
$$




\section{References}

[1] A.H. Nayfeh, D.T. Mook, S. Sridhar, Nonlinear analysis of the forced response of structural elements, J. Acoust. Soc. Am. 55 (2) (1974) $281-291$.

[2] D.T. Mook, A.H. Nayfey, Nonlinear Oscillations, Wiley-Interscience, 1979.

[4] T.M. Cameron, J.H. Griffin, P. Grant, An alternating frequency time domain method for calculating the steady state response of nonlinear dynamic systems, J. Appl. Mech. 56 (1) (1989) 149-154.

[5] D. Charleux, Etude des effets de la friction en pied d'aube sur la dynamique des roues aubagées, Ph.D. Thesis, Ecole Centrale Lyon, 2006.

[6] D. Charleux, F. Thouverez, J.P. Lombard, Three dimensional multiharmonic analysis of contact and friction in dovetail joints, in: IMAC XXII, Dearborn, Michigan, Paper 348, 2004

[7] E. Sarrouy, F. Thouverez, Global search of nonlinear system periodic solutions: a rotor dynamic application, Mech. Syst. Signal Process. 24 (2010) 1799-1813.

[8] K. Ekici, K.C. Hall, E.H. Dowell, Computationally fast harmonic balance methods for unsteady aerodynamic predictions of helicopter rotors, J. Comput. Phys. 227 (12) (2008) 6206-6225.

[9] G. Kerschen, M. Peeters, J.C. Golinval, A.F. Vakakis, Nonlinear normal modes, part i: a useful framework for the structural dynamicist, Mech. Syst. Signal Process. 23 (2009) 170-194.

[10] G. Groll, D.J. Ewins, The harmonic balance method with arc-length continuation in rotor stator contact problems, J. Sound Vibr. 241 (2) (2001) 223-233.

[11] V. Jaumouillé, J.J. Sinou, B. Petitjean, An adaptive harmonic balance method for predicting the nonlinear dynamic responses of mechanical systems-application to bolted structures, J. Sound Vibr. 329 (19) (2010) 4048-4067.

[12] D. Laxalde, Etude d'amortisseur non linéaires appliqués aux roue aubagées et au systèmes multi-étages, Ph.D. Thesis, Ecole Centrale Lyon, 2007.

[13] R.C. Maple, P.I. King, P.D. Orkiwis, J.M. Wolff, Adaptive harmonic balance method for nonlinear time periodic flows, J. Comput. Phys. 193 (2004) 620-641.

[14] R.C. Maple, P.I. King, M.E. Oxley, Adaptive harmonic balance solutions to Eulerś equation, AIAA J. 41 (9) (2003).

[15] A.H. Nayfey, B. Balanchandran, Applied Nonlinear Dynamics, Wiley-Interscience, 1995.

[16] P. Ribiero, M. Petyt, Geometrical non linear, steady state, forced periodic vibration of plates, part i: model and convergence studies, J. Sound Vibr. 226 (5) (1999) 938-955.

[17] L. Salles, L. Blanc, F. Thouverez, A.M. Gouskov, Dynamic analysis of a bladed disk with friction and fretting-wear in blade attachments, in: ASME Conference Proceedings, 2009, pp. 465-476. 